\title{
Post-Minkowskian effective field theory for conservative binary dynamics
}

\author{
Gregor Kälin ${ }^{a, b}$ and Rafael A. Porto ${ }^{b}$ \\ ${ }^{a} S L A C$ National Accelerator Laboratory, Stanford University, \\ Stanford, CA 94309, U.S.A. \\ ${ }^{b}$ Deutsches Elektronen-Synchrotron DESY, \\ Notkestrasse 85, 22607 Hamburg, Germany \\ E-mail: greka@slac.stanford.edu, rafael.porto@desy.de
}

ABstract: We develop an Effective Field Theory (EFT) formalism to solve for the conservative dynamics of binary systems in gravity via Post-Minkowskian (PM) scattering data. Our framework combines a systematic EFT approach to compute the deflection angle in the PM expansion, together with the 'Boundary-to-Bound' (B2B) dictionary introduced in $[1,2]$. Due to the nature of scattering processes, a remarkable reduction of complexity occurs both in the number of Feynman diagrams and type of integrals, compared to a direct EFT computation of the potential in a PM scheme. We provide two illustrative examples. Firstly, we compute all the conservative gravitational observables for bound orbits to $2 \mathrm{PM}$, which follow from only one topology beyond leading order. The results agree with those in $[1,2]$, obtained through the 'impetus formula' applied to the classical limit of the one loop amplitude in Cheung et al. [3]. For the sake of comparison we reconstruct the conservative Hamiltonian to $2 \mathrm{PM}$ order, which is equivalent to the one derived in [3] from a matching calculation. Secondly, we compute the scattering angle due to tidal effects from the electric- and magnetic-type Love numbers at leading PM order. Using the B2B dictionary we then obtain the tidal contribution to the periastron advance. We also construct a Hamiltonian including tidal effects at leading PM order. Although relying on (relativistic) Feynman diagrams, the EFT formalism developed here does not involve taking the classical limit of a quantum amplitude, neither integrals with internal massive fields, nor additional matching calculations, nor spurious ('super-classical') infrared singularities. By construction, the EFT approach can be automatized to all PM orders.

KEYwords: Classical Theories of Gravity, Effective Field Theories, Scattering Amplitudes ARXIV EPRINT: 2006.01184 


\section{Contents}

1 Introduction 1

2 Boundary-to-bound $\quad 6$

2.1 From angles to action ... 6

2.2 ... to binary observables

2.3 Hamiltonian $\quad 7$

3 Post-Minkowskian effective theory $\quad 9$

3.1 Point-particle sources $\quad 9$

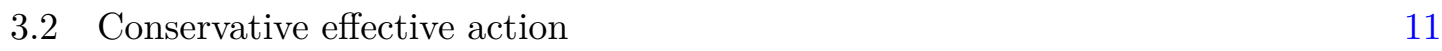

$\begin{array}{lll}3.3 & \text { Potential region } & 13\end{array}$

$\begin{array}{lll}3.4 & \text { Impulse \& deflection angle } & 14\end{array}$

4 Conservative binary dynamics to 2PM 16

4.1 Effective Lagrangian 16

$\begin{array}{lll}4.1 .1 & \text { Tree } & 16\end{array}$

$\begin{array}{lll}4.1 .2 & \text { One loop } & 17\end{array}$

$\begin{array}{lll}\text { 4.1.3 Trajectories } & 17\end{array}$

$\begin{array}{lll}4.2 & \text { Scattering angle } & 18\end{array}$

$\begin{array}{lll}\text { 4.2.1 Leading order impulse } & 18\end{array}$

$\begin{array}{ll}\text { 4.2.2 Next-to-leading order impulse } & 19\end{array}$

$\begin{array}{lll}4.3 & \text { Adiabatic invariants } & 23\end{array}$

4.3.1 Circular orbits 24

4.4 Hamiltonian 25

$5 \quad$ Leading tidal effects $\quad 25$

6 Discussion \& outlook $\quad 27$

A Action \& impulse vs. amplitude \& eikonal 30

\section{Introduction}

The discovery potential in gravitational wave $(\mathrm{GW})$ science $^{1}[4]$ relies in our ability to make precise theoretical predictions [5-7]. While the late stages of the binary dynamics require numerical modeling, the majority of GW cycles occur during the inspiral regime where perturbative approximations to Einstein's equations, such as the Post-Newtonian (PN) expansion, remain of vital importance to provide a faithful reconstruction of the signal [5].

\footnotetext{
${ }^{1}$ https://www.gw-openscience.org.
} 
It is in this regime where the effective field theory (EFT) approach introduced in [8], aka Non-Relativistic General Relativity (NRGR), has proven to be very successful to tackle the binary problem in gravity, see e.g. [9-14] for various reviews. In addition to the systematization of the problem of motion into the computation of a series of Feynman diagrams, and naturally incorporating finite-size effects via worldline terms beyond minimal coupling, one of the main virtues of the EFT formalism is the use of the method of regions [15], which allows us to disentangle the relevant physics involving both (off-shell) potential and (on-shell) radiation modes. The EFT methodology not only allows us to separate the computation of the relevant ingredients for waveform modeling into the 'conservative' and 'radiative' sectors, respectively, it also naturally handles the spurious infrared (IR) and ultraviolet (UV) divergences that appear from simplifying the resulting integrals by splitting into regions [16, 17], as well as the UV divergences due to the use of localized sources [8]. As a result, joining an effort which involves also more 'traditional' methods [18-24], the present state-of-the-art in NRGR is at the fourth PN (4PN) order of accuracy in the conservative sector for non-spinning bodies [8, 17, 25-31], which includes also contributions induced from (conservative) radiation-reaction effects $[16,17,28] .{ }^{2}$ This represents the next-tonext-to-next-to-next-to leading order $\left(\mathrm{N}^{4} \mathrm{LO}\right)$, or 'four loops', level of precision. Partial results have also been computed in NRGR at higher orders, both in the static sector at $5 \mathrm{PN}[52,53]$ and for hereditary effects at NLO [54, 55].

The method of regions is one of the trademarks of the EFT framework in a PN regime, allowing us to expand integrals in the potential region $\left(k^{0} \ll|\boldsymbol{k}|\right)$ in powers of $k_{0} /|\boldsymbol{k}|$. At the level of the computation of the gravitational potential, this is the reason for the (in)famous IR divergences appearing at 4PN, that ultimately cancel out in NRGR once conservative effects from radiation-reaction are incorporated $[16,17,28,31] .^{3}$ Furthermore, all the velocity (relativistic) corrections, both from vertex and propagator corrections, are truncated depending on the factors of Newton's constant $(G)$ in each given contribution, rather than incorporated to all orders from the onset. The reason being due to the virial theorem, relating $G M / r \sim v^{2}$ for bound states, which implies that only terms scaling as $G^{\ell} v^{2(n-\ell)}$ (with $\ell \leq n$ ) are needed to $n \mathrm{PN}$ order. While some of the factors of $k_{0}$, from the expansion of the Green's functions in NRGR, wound up producing acceleration-dependent contributions to the gravitational potential which can be traded for higher powers of $G$ using lower order equations of motion, an infinite series of velocity corrections is truncated. Therefore, although the expansion in the potential region is extremely powerful, reducing complex four- into three-dimensional massless integrals, e.g. [29], the natural expansion of field theory in powers of the coupling, or the Post-Minkowskian (PM) expansion in this context, begs us for a framework in which (special) relativistic effects are included to all orders.

\footnotetext{
${ }^{2}$ Radiation effects can be systematically incorporated in NRGR in terms of source and radiative multipoles, see e.g [32-36]. Moreover, spinning compact objects were introduced in [37] and also extensively studied in the NRGR literature, see e.g. [38-51]. We will study both radiation and rotation in future work.

${ }^{3}$ The IR divergences led to the introduction of ambiguity-parameters in other approaches [20-23], yet to be resolved within the PN regime in the ADM formalism of [19]. See [24] for an ambiguity-free derivation following dimensional regularization, as advocated in $[17,28]$, including a re-derivation of the conservative tail effect computed in [28].
} 
An obvious option to implement a PM expansion for bound orbits is to resum all the velocity corrections in the NRGR computation of the potential to a given order in $G$, while dropping terms depending on the accelerations. This, however, turns out to be unnecessarily cumbersome. (See [56] for an attempt at 1PM.) Moreover, since the potential is a gauge-dependent quantity, many (many) different terms can be present depending on the gauge, with Lorentz invariance only manifest in the final expressions. Another option is to attempt to perform the much more difficult exact relativistic integration, keeping only real contributions to the effective potential. As we shall see, however, a simpler procedure can be adopted, in which rather than bringing the PM expansion to NRGR, we will bring instead the EFT approach to the natural habitat of the PM framework. Inspired by the study of relativistic scattering, in this paper we implement an EFT formalism to compute the deflection angle perturbatively in $G$, but to all orders in the relative velocity. Even though, in principle, it may seem like scattering processes are unrelated to bound orbits, a remarkable connection has been recently uncovered in $[1,2]$, in the form of a 'Boundary-toBound' (B2B) dictionary. The map put forward in [1, 2] relates scattering data directly to dynamical invariants for binary systems, without using a Hamiltonian. Our goal in this paper, as suggested also in $[1,2]$, is therefore to combine the virtues of the EFT formalism and B2B dictionary $[1,2]$ to construct a systematic EFT approach to the conservative dynamics of binary systems in the PM expansion, without ever resorting to gauge-dependent objects.

The idea of mapping (quantum) scattering information into the (classical) physics of binary systems, dating back to early efforts in the 70's, e.g. [57], has been reinvigorated by the recent program to connect amplitudes techniques from high-energy physics [58-61] to the derivation of the two-body gravitational dynamics in the classical regime, e.g. [3, 6291]. Furthermore, the connection between scattering data and binary dynamics has also been emphasized in the context of the effective one body (EOB) approach [92], e.g. [93101]. However, in all these cases the derivation of a gauge-dependent and rather lengthy Hamiltonian, or EOB equivalent, has played a central role. This feature was, after all, one of the main reasons that motivated us in $[1,2]$ to construct the B2B map directly between the (much simpler) gauge-invariant observables. The B2B dictionary was originally introduced in [1], via the construction of a radial action depending on the analytic continuation of scattering data. Although the radial action was first discussed in the context of the PN expansion, see e.g. [102], the analysis in [1, 2] unveiled for the first time the astonishingly simple structure in the PM framework instead. In its first incarnation, the B2B radial action was built upon a remarkable connection between the analytic continuation of the scattering amplitude in the classical limit and the momentum of the particles in the center-of-mass frame, which we dubbed the 'impetus formula' [1]. We later extended the dictionary in [2], unraveling an unexpected relationship between the deflection angle and periastron advance, once again via analytic continuation, which allowed us to reconstruct the B2B map entirely in terms of the scattering angle without invoking the impetus formula. The analysis in $[1,2]$ has thus uncovered a surprisingly simple hidden structure of the radial action in the PM conservative sector, readily mapping scattering (boundary) data to dynamical invariants for generic (bound) orbits. While the B2B dictionary neatly translates all the gauge-invariant scattering information into observables for binary systems, it has been implemented so 
far via the classical limit of the amplitude, either through the impetus formula or from the scattering angle, computed in $[63,64]$ to $3 \mathrm{PM}$ order (or two loops). Motivated by the prowesses of NRGR, in this paper we provide an alternative framework to collect the necessary scattering data to input in the B2B dictionary, using instead an EFT approach adapted to the computation of the impulse and scattering angle in the PM scheme.

The powerful formalism developed in $[3,63,64]$ has demonstrated its ability to obtain physical information from scattering amplitudes to high PM orders. Yet, in its present form, it relies on taking the classical limit of a quantum amplitude, performing a matching calculation to an effective theory built with a local potential interaction and, perhaps more importantly, relativistic integrals involving internal massive propagators yielding spurious IR divergences. The singular terms, due to so-called 'super-classical' contributions, ultimately cancel out either during the matching calculation in $[3,63,64]$ or equivalent after including 'Born iterations' $[68,77,86]$. However, they signal a generic feature of computations involving the classical limit of quantum amplitudes. ${ }^{4}$ Hence, while the method of generalized unitarity $[103,104]$ and the double copy technique $[105,106]$ allow to bypass the need of a large set of Feynman diagrams [64], ${ }^{5}$ and the impetus formula [1] can sidestep the need of a matching calculation to extract the Hamiltonian, it is still worthwhile to develop an independent systematic framework to compute classical observables for bound orbits from scattering data, without the type of integrals and subtle limits that appear in the amplitude program.

In an EFT approach to gravity for extended objects, the bodies are treated as external localized sources endowed with a series of (Wilson) coefficients that parameterize finitesize effects [8]. Therefore, we do not include mass-dependent propagators, and as long as loops of the gravitational field are avoided, we always remain in the classical realm. As a result, the derivation of the scattering angle is reduced into two- and three-dimensional integrals involving only massless propagators. Not only these are easier to compute, we do not encounter spurious super-classical IR singularities, such as e.g. the box diagram at one loop $[3,77] .{ }^{6}$ There are, however, various similarities between the approach developed in $[3,63,64]$ and the one introduced here. For instance, one of the key aspects of our formalism consists on isolating the conservative contribution from the potential region. This is achieved by providing a prescription to perform the energy integrals, which turns out to be similar to the one in $[3,63,64]$, except for the (lack of) 'anti-matter' poles and massive internal fields. Moreover, as we shall see, cancelations also occur in our derivation of the scattering angle. For example, the vanishing of a term which we may identify with the crossed-box diagram in [64].

\footnotetext{
${ }^{4}$ This issue is present also when dealing directly with gauge-invariant quantities, as shown in [65, 66] for the derivation of the scattering angle. In such case the cancelation occurs instead between two independent contributions to the impulse, linear and quadratic in the amplitude.

${ }^{5}$ The gravitational scattering amplitude at two loops has been recently computed using standard Feynman techniques in [107] and shown to agree with the results of [3, 63, 64].

${ }^{6}$ As we mentioned, other type of spurious divergences may appear when dealing with potential modes [17, 28, 31]. However, we expect these to explicitly cancel out in a fully relativistic framework that also incorporates conservative radiation-reaction effects. We will return to this in future work.
} 
An EFT approach for the PM expansion has many virtues, yet the disadvantage with respect to the amplitudes program of $[3,63,64]$ is the persistent reliance on the perturbative machinery of Feynman diagrams, when it comes to purely classical computations. However, because of the notorious simplifications of the scattering process, even at the classical level, a comparatively small number of diagrams are needed, significantly reducing the amount of combinatorial complexity. For instance, only two are required to 2PM, which we compute in detail in this paper, whereas only an additional five enter in the derivation to 3PM order, reported elsewhere [108]. Therefore, the EFT formalism in the PM scheme provides an alternative systematic machinery to compute the PM conservative dynamics of binary systems, potentially reaching the same level of success as NRGR in the PN expansion - currently at $\mathrm{N}^{4} \mathrm{LO}$ (akin of 5PM accuracy) - using the existent technology in the field [30, 31], and elsewhere [109]. Yet, since the number of diagrams and necessary steps will at some point start to escalate quickly, it is expected that the double copy and other on-shell techniques from the amplitude program may be able to reduce further the level of complexity in the derivation of the scattering angle. (Another possibility is to recast Einstein's gravity as in [110], to simplify the number of Feynman diagrams.) Unfortunately, at the moment, we have not found a simple way to incorporate these tools into the EFT formalism, although ideas from the classical doubly copy, e.g. [111-121], may ultimately provide a hybrid framework to march smoothly towards higher orders.

This paper is organized as follows. In section 2 we review the B2B map, emphasizing the construction of the radial action from PM scattering data. In section 3 we introduce the effective theory for conservative dynamics, the worldline and bulk action, as well as the integration prescription for potential modes. We then show how to use the effective theory to compute the impulse and deflection angle to all PM orders. In section 4 we demonstrate the power of the effective theory by (re)deriving the impulse and scattering angle to $2 \mathrm{PM}$ in a few simple steps, from which we obtain all the dynamical invariants for binary system through the B2B dictionary. For the sake of comparison, we reconstruct a 2PM Hamiltonian for the two-body dynamics, which we show is equivalent to the one computed in $[3,63,64]$. In section 5 we compute the leading PM contribution to the scattering angle from electricand magnetic-type tidal effects, which are obtained from insertions of higher dimensional operators in the worldline action. Following the B2B map we then obtain the leading correction to the periastron advance due to (spin-independent) finite-size effects at leading PM order. We also construct the associated Hamiltonian including tidal effects. We conclude with a summary and the road ahead in section 6 , and a few comment on the relationship between the action \& impulse versus the amplitude \& eikonal in the classical limit, in appendix A.

Conventions. We use the mostly minus signature: $\eta_{\mu \nu}=\operatorname{diag}(+,-,-,-)$. The Minkowski product between four-vectors is denoted as $k \cdot x=\eta_{\mu \nu} k^{\mu} x^{\nu}$, while we use $\boldsymbol{k} \cdot \boldsymbol{x}=\delta^{i j} \boldsymbol{k}^{i} \boldsymbol{x}^{j}$ for the Euclidean version, with bold letters representing 3 -vectors. We use $\boldsymbol{k}_{\perp}$ for vectors in the plane perpendicular to the direction of the scattering particles. We use the shorthand notation $\int_{k} \equiv \int d^{4} k /(2 \pi)^{4}, \int_{\boldsymbol{k}} \equiv \int d^{3} \boldsymbol{k} /(2 \pi)^{3}$, and $\int_{\boldsymbol{k}_{\perp}} \equiv \int d^{2} \boldsymbol{k}_{\perp} /(2 \pi)^{2}$. We also absorb factors of $2 \pi$ into the $\delta$ functions: $\hat{\delta}(x) \equiv 2 \pi \delta(x)$. For divergent integrals we use dimensional regularization, such that the number of dimensions is replaced by $D=d-2 \epsilon$, 
with $d$ either 4,3 or 2 . We use $M_{\mathrm{Pl}}^{-1} \equiv \sqrt{32 \pi G}$ for the Planck mass in $\hbar=c=1$ units. We denote $M=m_{1}+m_{2}$ the total mass, $\mu=m_{1} m_{2} / M$ the reduced mass, and $\nu \equiv \mu / M$ the symmetric mass ratio.

\section{Boundary-to-bound}

In this section we briefly review the ingredients introduced in $[1,2]$ to compute gravitational observables for binary systems using scattering data. For the sake of comparison, we also illustrate the reconstruction of the Hamiltonian from the scattering angle.

\subsection{From angles to action ...}

In this paper we will not use the impetus formula introduced in the B2B map of [1] to construct the radial action. Instead, we use the representation discovered in [2] from the relationship between the scattering angle and periastron advance, yielding

$$
i_{r} \equiv \frac{\mathcal{S}_{r}}{G M \mu}=\frac{\hat{p}_{\infty}}{\sqrt{-\hat{p}_{\infty}^{2}}} \chi_{j}^{(1)}-j\left(1+\frac{2}{\pi} \sum_{n=1} \frac{\chi_{j}^{(2 n)}}{(1-2 n) j^{2 n}}\right)
$$

The PM coefficients of the scattering angle are defined through

$$
\frac{\chi}{2}=\sum_{n=1} \chi_{j}^{(n)} / j^{n}
$$

with $j=J /(G M \mu)$ the reduced angular momentum. The energy of the two-body system can be written as

$$
E=M+\mu \mathcal{E}=M(1+\nu \mathcal{E}),
$$

with the momentum at infinity (in the center-of-mass frame) given by

$$
p_{\infty}=\mu \frac{\sqrt{\gamma^{2}-1}}{\Gamma}=\mu \hat{p}_{\infty}
$$

with

$$
\begin{aligned}
\gamma & \equiv \frac{p_{1} \cdot p_{2}}{m_{1} m_{2}}=u_{1} \cdot u_{2}=\frac{E^{2}-m_{1}^{2}-m_{2}^{2}}{2 m_{1} m_{2}}=1+\mathcal{E}+\frac{\nu \mathcal{E}^{2}}{2}, \\
\Gamma & \equiv E / M=\sqrt{1+2 \nu(\gamma-1)} .
\end{aligned}
$$

The representation in angular-momentum space may be obtained from the expansion in impact parameter,

$$
\frac{\chi}{2}=\sum_{n=1} \chi_{b}^{(n)}\left(\frac{G M}{b}\right)^{n}
$$

by replacing $j^{-1}=\hat{p}_{\infty}^{-1} \frac{G M}{b}$ in $(2.2)$ such that $\chi_{j}^{(n)}=\hat{p}_{\infty}^{n} \chi_{b}^{(n)}$. 


\section{$2.2 \quad \ldots$ to binary observables}

Once the radial action is reconstructed from scattering data, and after analytic continuation to negative binding energies, $\mathcal{E}<0$, the gravitational observables are obtained via differentiation. For instance, the periastron advance and periastron-to-periastron period are given by $[1,2]$

$$
\frac{\Delta \Phi(j, \mathcal{E})}{2 \pi}=-\frac{\partial}{\partial j}\left(i_{r}+j\right)=\frac{1}{\pi} \sum_{n=1} \frac{2 \chi_{j}^{(2 n)}(\mathcal{E})}{j^{2 n}}=\frac{1}{2 \pi}(\chi(j, \mathcal{E})+\chi(-j, \mathcal{E})),
$$

and

$$
\frac{T_{p}}{2 \pi}=G M \frac{\partial}{\partial \mathcal{E}} i_{r}(j, \mathcal{E})=G E \frac{\partial}{\partial \gamma} i_{r}(j, \gamma),
$$

respectively. From here we can obtain the azimuthal and radial frequencies, defined through

$$
\begin{aligned}
\Omega_{r}(j, \mathcal{E}) & \equiv \frac{2 \pi}{T_{p}}, \quad \Omega_{p}(j, \mathcal{E}) \equiv \frac{\Delta \Phi}{T_{p}}, \\
\Omega_{\phi} & \equiv \Omega_{r}+\Omega_{p}=\frac{2 \pi}{T_{p}}\left(1+\frac{\Delta \Phi}{2 \pi}\right) .
\end{aligned}
$$

We can also compute the redshift function, $\left\langle z_{a}\right\rangle$, using the first-law [122], yielding [2]

$$
\delta \mathcal{S}_{r}\left(J, \mathcal{E}, m_{a}\right)=-\left(1+\frac{\Delta \Phi}{2 \pi}\right) \delta J+\frac{\mu}{\Omega_{r}} \delta \mathcal{E}-\sum_{a} \frac{1}{\Omega_{r}}\left(\left\langle z_{a}\right\rangle-\frac{\partial E\left(\mathcal{E}, m_{a}\right)}{\partial m_{a}}\right) \delta m_{a} .
$$

\subsection{Hamiltonian}

The construction of the B2B radial action allows us to bypass the need of a Hamiltonian, or the very equations of motion, to derive all the gravitational observables for the binary system in the conservative sector. It is possible, however, to reconstruct a Hamiltonian from which these observables may also be computed. The procedure was described in [1], and relies on Firsov's solution to the scattering problem [123]. It starts by solving for the $f_{n}$ coefficients in the PM expansion of the (square of the) momentum in the center-of-mass frame,

$$
\boldsymbol{p}^{2}=p_{\infty}^{2}\left(1+\sum_{n=1} f_{n}\left(\frac{G M}{r}\right)^{n}\right)
$$

as a function of the scattering angle. The connection, as well as the inverse formula, was unfolded in [1] to all orders in $G$. It reads

$$
f_{n}=\sum_{\sigma \in \mathcal{P}(n)} g_{\sigma}^{(n)} \prod_{\ell}\left(\widehat{\chi}_{b}^{\left(\sigma_{\ell}\right)}\right)^{\sigma^{\ell}}
$$

where

$$
\widehat{\chi}_{b}^{(n)} \equiv \frac{2}{\sqrt{\pi}} \frac{\Gamma\left(\frac{n}{2}\right)}{\Gamma\left(\frac{n+1}{2}\right)} \chi_{b}^{(n)},
$$

and $\mathcal{P}(n)$ is the set of all integer partitions of $n$. Each partition is described by $n=\sigma_{\ell} \sigma^{\ell}$ (implicit summation) with mutually different $\sigma_{\ell}$ 's. Introducing the notation $\Sigma^{\ell} \equiv \sum_{\ell} \sigma^{\ell}$, the coefficients are given by

$$
g_{\sigma}^{(n)}=\frac{2(2-n)^{\Sigma^{\ell}-1}}{\prod_{\ell}\left(2 \sigma^{\ell}\right) ! !} .
$$


We can also invert the relation in (2.13), solving for the scattering angle as a function of the momentum, yielding

$$
\chi_{b}^{(n)}=\frac{\sqrt{\pi}}{2} \Gamma\left(\frac{n+1}{2}\right) \sum_{\sigma \in \mathcal{P}(n)} \frac{1}{\Gamma\left(1+\frac{n}{2}-\Sigma^{\ell}\right)} \prod_{\ell} \frac{f_{\sigma_{\ell}}^{\sigma^{\ell}}}{\sigma^{\ell !}} .
$$

See [1] for more details. Once the $f_{n}$ 's are known, we can reconstruct a Hamiltonian as follows. For convenience, we write the expressions in terms of $P_{n} \equiv p_{\infty}^{2} M^{n} f_{n}$, which is more suitable to define the gravitational potential. We begin with the equation for $E$ as a function of $p_{\infty}^{2}$,

$$
E=\sqrt{p_{\infty}^{2}+m_{1}^{2}}+\sqrt{p_{\infty}^{2}+m_{2}^{2}},
$$

which we then use to construct a Hamiltonian in 'isotropic' gauge, defined as

$$
E=H\left(r, \boldsymbol{p}^{2}\right)=\sum_{i=0}^{\infty} \frac{c_{i}\left(\boldsymbol{p}^{2}\right)}{i !}\left(\frac{G}{r}\right)^{i}
$$

in the PM expansion. (Notice we use a slightly different normalization than [3, 63, 64] for the $c_{i}$ coefficients.) Using (2.12) we have the condition

$$
\sqrt{\boldsymbol{p}^{2}-\sum_{i=1}^{\infty} P_{i}(E)\left(\frac{G}{r}\right)^{i}+m_{1}^{2}}+\sqrt{\boldsymbol{p}^{2}-\sum_{i=1}^{\infty} P_{i}(E)\left(\frac{G}{r}\right)^{i}+m_{2}^{2}}=\sum_{i=0}^{\infty} \frac{c_{i}\left(\boldsymbol{p}^{2}\right)}{i !}\left(\frac{G}{r}\right)^{i}
$$

that can be solved iteratively for the $c_{i}$ 's in terms of recursive relations. At zeroth order we have:

$$
c_{0}\left(\boldsymbol{p}^{2}\right)=E\left(\boldsymbol{p}^{2}\right)=E_{1}\left(\boldsymbol{p}^{2}\right)+E_{2}\left(\boldsymbol{p}^{2}\right) \equiv \sqrt{\boldsymbol{p}^{2}+m_{1}^{2}}+\sqrt{\boldsymbol{p}^{2}+m_{2}^{2}},
$$

and up to $2 \mathrm{PM}$ order,

$$
\begin{aligned}
c_{1}\left(\boldsymbol{p}^{2}\right)= & -\frac{1}{2 E \xi} P_{1}(E)=-\frac{\nu^{2} M^{2}}{\xi \Gamma^{2}} \frac{\left(\gamma^{2}-1\right) \chi_{b}^{(1)}(E)}{\Gamma} \\
c_{2}\left(\boldsymbol{p}^{2}\right)= & -\frac{1}{E \xi} P_{2}(E)+\frac{(3 \xi-1) P_{1}^{2}(E)}{4 E^{3} \xi^{3}}+\frac{P_{1}(E) P_{1}^{\prime}(E)}{2 E^{2} \xi^{2}} \\
= & -\frac{\nu^{2} M^{3}}{\xi \Gamma^{2}}\left(\frac{4\left(\gamma^{2}-1\right)}{\pi} \frac{\chi_{b}^{(2)}(E)}{\Gamma}-\frac{(3 \xi-1) \nu^{2}}{\xi^{2} \Gamma^{3}}\left(\left(\gamma^{2}-1\right) \frac{\chi_{b}^{(1)}(E)}{\Gamma}\right)^{2}\right. \\
& \left.-\frac{2 \nu^{2}\left(\gamma^{2}-1\right)}{\Gamma \xi} \frac{\chi_{b}^{(1)}(E)}{\Gamma} \frac{d}{d E}\left(\frac{E\left(\gamma^{2}-1\right) \chi_{b}^{(1)}(E)}{\Gamma^{3}}\right)\right)
\end{aligned}
$$

where $\xi(\boldsymbol{p}) \equiv E_{1}(\boldsymbol{p}) E_{2}(\boldsymbol{p}) / E^{2}(\boldsymbol{p})$. We have written the coefficients directly in terms of the scattering angle as a function of the energy to 2PM. At the end of the day, all the functions must be understood as defined in terms of the momentum through $E(\boldsymbol{p})$ in (2.20). General expressions to all orders can be found in [1]. 


\section{Post-Minkowskian effective theory}

In this section we develop an EFT approach to obtain the impulse and scattering angle in the PM framework. We provide a set of simplified Feynman rules for the Einstein-Hilbert and worldline action and discuss the computation of the conservative contributions from potential modes as well as the required integration procedure.

\subsection{Point-particle sources}

Following the EFT approach developed in [8], we construct a worldline action to describe both constituents of the two-body problem,

$$
S_{\mathrm{pp}}=-\sum_{a} m_{a} \int \mathrm{d} \sigma_{a} \sqrt{g_{\mu \nu}\left(x_{a}^{\alpha}(\sigma)\right) v_{a}^{\mu}\left(\sigma_{a}\right) v_{a}^{\nu}\left(\sigma_{a}\right)}+\cdots,
$$

where $v^{\mu}=\frac{d x^{\mu}}{d \sigma}$, and the ellipses account for finite-size effects (as well as counter-terms). For instance, using the proper time, $\tau$, we have

$$
\cdots=\int \mathrm{d} \tau_{a}\left(c_{R}^{(a)} R\left(x_{a}\right)+c_{V}^{(a)} R_{\mu \nu}\left(x_{a}\right) v_{a}^{\mu} v_{a}^{\nu}+c_{E^{2}}^{(a)} E_{\mu \nu}\left(x_{a}\right) E^{\mu \nu}\left(x_{a}\right)+c_{B^{2}}^{(a)} B_{\mu \nu}\left(x_{a}\right) B^{\mu \nu}\left(x_{a}\right) \ldots\right),
$$

and additional operators can be added to include spin effects [14]. The $c_{R, V}$ coefficients do not contribute to physical quantities, since they can be removed by field redefinitions [8], however they may be needed to properly renormalize the theory removing intermediate UV poles [31]. The $c_{E^{2}}$ and $c_{B^{2}}$ operators, written in terms of the electric and magnetic components of the Weyl tensor, represent the tidal Love number (which vanish for nonrotating black holes [6]). We will study later on the leading contribution from these tidal operators to the scattering angle in section 5 . Other terms can be systematically included in a derivative expansion [14]. Notice that the action is reparameterization invariant. This allows us to fix the gauge, for instance with the coordinate time $x^{0}=\sigma$, which is useful in the PN expansion.

In the PM scheme, instead of (3.1) it is convenient to work with the Polyakov action, ${ }^{7}$

$$
S_{\mathrm{pp}}=-\sum_{a=1,2} \frac{m_{a}}{2} \int \mathrm{d} \sigma_{a} e_{a}\left(\frac{1}{e_{a}^{2}} g_{\mu \nu}\left(x_{a}^{\alpha}(\sigma)\right) v_{a}^{\mu}\left(\sigma_{a}\right) v_{a}^{\nu}\left(\sigma_{a}\right)+1\right),
$$

such that variations with respect to $e_{a}$ give $e_{a}=\sqrt{g_{\mu \nu} v_{a}^{\mu} v_{a}^{\nu}}$, thus recovering (3.1). The advantage of this action is that, in contrast to PN computations, the PM approximation is suited for using the proper, rather than coordinate, time to parameterize the trajectories. Therefore we will choose the gauge in which $e_{a}=1$, yielding $\sigma_{a}=\tau_{a}$. The worldline action becomes simply

$$
S_{\mathrm{pp}}=-\sum_{a} \frac{m_{a}}{2} \int \mathrm{d} \tau_{a} g_{\mu \nu}\left(x_{a}\left(\tau_{a}\right)\right) v_{a}^{\mu}\left(\tau_{a}\right) v_{a}^{\nu}\left(\tau_{a}\right) .
$$

with the condition

$$
e_{a}^{2}=g_{\mu \nu}\left(x_{a}\left(\tau_{a}\right)\right) v_{a}^{\mu}\left(\tau_{a}\right) v_{a}^{\nu}\left(\tau_{a}\right)=1 .
$$

\footnotetext{
${ }^{7}$ The Polyakov action may also be useful to study the high-energy limit of the self-force problem [67], as well as to simplify PN computation [124] using the coordinate time.
} 
From (3.4) the geodesic equation may be written in compact form as

$$
\frac{d}{d \tau_{a}}\left(g_{\alpha \mu}\left(x_{a}\left(\tau_{a}\right)\right) v_{a}^{\mu}\left(\tau_{a}\right)\right)=\frac{1}{2} \frac{\partial g_{\mu \nu}}{\partial x^{\alpha}}\left(x_{a}\left(\tau_{a}\right)\right) v_{a}^{\mu}\left(\tau_{a}\right) v_{a}^{\nu}\left(\tau_{a}\right),
$$

although in the EFT formalism we will work directly with the effective Lagrangian instead. Notice that, expanding the metric in the weak field approximation,

$$
g_{\mu \nu}=\eta_{\mu \nu}+\frac{h_{\mu \nu}}{M_{\mathrm{Pl}}}
$$

the action in (3.4) only generates a one-point function. In turn, all the non-linear effects are therefore encoded in the bulk action. We will work in Einstein's gravity, described by the Einstein-Hilbert action

$$
S_{\mathrm{EH}}=-2 M_{\mathrm{Pl}}^{2} \int \mathrm{d}^{4} x \sqrt{-g} R[g] .
$$

It is advantageous to optimize the Feynman rules given by this action to obtain the fewest number of terms for each bulk vertex. For this purpose we use a generalized gauge-fixing condition, as well as judiciously chosen total derivatives. In principle, one can also use field redefinitions [125]. However, field redefinitions modify the worldline vertices introducing more diagrams. In this paper we choose to keep the worldline coupling unmodified, as in (3.4).

At quadratic order we retain the De-Donder gauge, and the two-point Lagrangian

$$
\mathcal{L}_{h h}=\frac{1}{2} \partial_{\alpha} h^{\mu \nu} \partial^{\alpha} h_{\mu \nu}-\frac{1}{4} \partial_{\mu} h \partial^{\mu} h,
$$

with $h \equiv h_{\alpha}^{\alpha}$. From here we find the propagator

$$
\left\langle h_{\mu \nu}(x) h_{\alpha \beta}(y)\right\rangle=\frac{i}{k^{2}} P_{\mu \nu \alpha \beta} e^{i k \cdot(x-y)},
$$

where (in $D$ dimensions)

$$
P_{\mu \nu \alpha \beta}=\frac{1}{2}\left(\eta_{\mu \alpha} \eta_{\nu \beta}+\eta_{\nu \alpha} \eta_{\mu \beta}-\frac{2}{D-2} \eta_{\mu \nu} \eta_{\alpha \beta}\right)
$$

For the three-point function, the shortest expression that does not modify the worldline coupling contains six terms,

$$
\begin{aligned}
M_{\mathrm{Pl}} \mathcal{L}_{h h h}= & -\frac{1}{2} h^{\mu \nu} \partial_{\mu} h^{\rho \sigma} \partial_{\nu} h_{\rho \sigma}+\frac{1}{2} h^{\mu \nu} \partial_{\rho} h \partial^{\rho} h_{\mu \nu}-\frac{1}{8} h \partial_{\rho} h \partial^{\rho} h \\
& +h^{\mu \nu} \partial_{\nu} h_{\rho \sigma} \partial^{\sigma} h_{\mu}{ }^{\rho}-h^{\mu \nu} \partial_{\sigma} h_{\nu \rho} \partial^{\sigma} h_{\mu}{ }^{\rho}+\frac{1}{4} h \partial_{\sigma} h_{\nu \rho} \partial^{\sigma} h^{\nu \rho}
\end{aligned}
$$

There exist other inequivalent three-point Lagrangians of the same size. The given representation was chosen such that $i$ ) it does not contain any double derivatives acting on the same field and $i i$ ) the four-point Lagrangian has the least amount of terms which is found 
to be

$$
\begin{aligned}
M_{\mathrm{Pl}}^{2} \mathcal{L}_{h h h h}= & -\frac{1}{4} h^{\mu \nu} h^{\rho \sigma} \partial_{\alpha} h_{\rho \sigma} \partial^{\alpha} h_{\mu \nu}+\frac{1}{2} h^{\mu \nu} h^{\rho \sigma} \partial_{\alpha} h_{\nu \sigma} \partial^{\alpha} h_{\mu \rho}+h_{\mu}{ }^{\rho} h^{\mu \nu} \partial_{\alpha} h_{\rho \sigma} \partial^{\alpha} h_{\nu}{ }^{\sigma} \\
& -\frac{1}{2} h h^{\nu \rho} \partial_{\alpha} h_{\rho \sigma} \partial^{\alpha} h_{\nu}{ }^{\sigma}-\frac{1}{8} h_{\mu \nu} h^{\mu \nu} \partial_{\alpha} h_{\rho \sigma} \partial^{\alpha} h^{\rho \sigma}+\frac{1}{16} h^{2} \partial_{\alpha} h_{\rho \sigma} \partial^{\alpha} h^{\rho \sigma} \\
& -\frac{3}{4} h^{\mu \nu} h^{\rho \sigma} \partial_{\nu} h_{\sigma \alpha} \partial_{\rho} h_{\mu}{ }^{\alpha}-h_{\mu}{ }^{\rho} h^{\mu \nu} \partial^{\alpha} h_{\nu}{ }^{\sigma} \partial_{\rho} h_{\sigma \alpha}+\frac{1}{2} h h^{\nu \rho} \partial^{\alpha} h_{\nu}{ }^{\sigma} \partial_{\rho} h_{\sigma \alpha} \\
& +\frac{1}{2} h_{\mu}{ }^{\rho} h^{\mu \nu} \partial_{\nu} h^{\sigma \alpha} \partial_{\rho} h_{\sigma \alpha}-\frac{1}{4} h^{\mu}{ }_{\mu} h^{\nu \rho} \partial_{\nu} h^{\sigma \alpha} \partial_{\rho} h_{\sigma \alpha}-\frac{1}{2} h^{\mu \nu} h^{\rho \sigma} \partial^{\alpha} h_{\mu \rho} \partial_{\sigma} h_{\nu \alpha} \\
& +h^{\mu \nu} h^{\rho \sigma} \partial_{\rho} h_{\mu}{ }^{\alpha} \partial_{\sigma} h_{\nu \alpha}+\frac{1}{4} h_{\mu}{ }^{\rho} h^{\mu \nu} \partial^{\alpha} h_{\nu}{ }^{\sigma} \partial_{\sigma} h_{\rho \alpha}-\frac{1}{2} h_{\mu}{ }^{\rho} h^{\mu \nu} \partial_{\sigma} h \partial^{\sigma} h_{\nu \rho} \\
& +\frac{1}{4} h h^{\nu \rho} \partial_{\sigma} h \partial^{\sigma} h_{\nu \rho}+\frac{1}{8} h_{\mu \nu} h^{\mu \nu} \partial_{\alpha} h_{\sigma}{ }^{\alpha} \partial^{\sigma} h^{\rho}{ }_{\rho}-\frac{1}{16} h^{2} \partial_{\alpha} h_{\sigma}{ }^{\alpha} \partial^{\sigma} h .
\end{aligned}
$$

Also for the four-point function there exist other versions of the same length.

The three-point vertex is all we need to $2 \mathrm{PM}$ (while the four-point vertex enters at 3PM), and therefore we find these rules more convenient than adding more topologies from non-linear couplings to the sources. However, at higher orders further simplifications can be achieved by allowing for field redefinitions. For instance, keeping the same propagator, the three- and four-point functions can be simplified to 3 and 12 terms respectively, at the cost of worldline non-linearities. (The cubic coupling with only two terms is also possible, resulting in three terms for the quadratic action and a modified propagator.) Other type of simplifications are in principle possible, for instance by recasting Einstein's gravity in terms of only cubic couplings [110], which can reduce the number of diagrams at higher PM orders.

\subsection{Conservative effective action}

Given the bulk and worldline action, we can 'integrate-out' the graviton field, namely the metric, to construct an effective two-body action. As in the original EFT approach $[8,14]$, we compute

$$
e^{i S_{\mathrm{eff}}\left[x_{a}\right]}=\int \mathcal{D} h_{\mu \nu} e^{i S_{\mathrm{EH}}[h]+i S_{\mathrm{GF}}[h]+i S_{\mathrm{pp}}\left[x_{a}, h\right]},
$$

where $S_{\mathrm{GF}}$ is the gauge fixing term we alluded before and we have omitted total derivatives we have used to simplify the Feynman rules. Field redefinitions of the metric field are also allowed, although we have not used them in the present paper. Despite the path integral representation, we use the saddle-point approximation keeping only the classical contributions to the effective action. We thus treat the massive particles as non-propagating external sources and include only connected tree level diagrams, i.e. without graviton loops. In other words, the path integral and associated Feynman rules are a convenient and systematic way to encapsulate solutions to Einstein's equations sourced by localized sources, which are then plugged back into the action. This procedure is sometimes referred in the literature as the construction of the 'Fokker-action', e.g. [18]. As in NRGR, the perturbative expansion in terms of iterated Green's functions will resemble loop-type integrals in field theory. Yet, no quantum effects are ever computed, although they can be easily incorporated [8]. The advantage of the EFT approach, in comparison with directly tackling Einstein's equations, is that we do not need to solve for the metric explicitly, which 


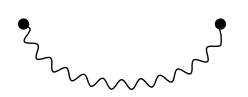

(a)

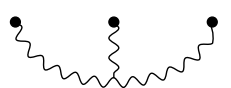

(b)

Figure 1. Feynman diagrams which only yield singular integrals in the potential region. The divergences can be set to zero in dimensional regularization, or absorbed into counter-terms.

is nonetheless unobservable in the conservative sector. Instead, we compute what is often described as the (connected) vacuum-to-vacuum amplitude in the presence of sources $\left({ }^{\prime}-i \log \langle 0 \mid 0\rangle^{J}\right.$ ') in the saddle-point approximation, simply steering away from closed quantum loops. In doing so, evaluating the effective action on solutions to the classical equations of motion, the power counting in Planck's constant becomes trivial and $\hbar$ turns into nothing but a conversion factor that drops out of the final answer.

In principle, the integrals that appear in the perturbative expansion of the path integral in (3.14) include contributions from two regions: potential and radiation modes [8]. The latter incorporate the propagating (on-shell) modes that travel to the gravitational wave detector, while the (off-shell) potential modes are responsible for the conservative forces that deflect the particles in a two-body encounter, and also bind the system together in a binary. In this paper we will only consider conservative effects. Since the imaginary part is due to cuts induced by radiation modes $[8,14]$, by concentrating in the conservative sector the effective action will remain real at all stages. As in any EFT without a cutoff in the momenta, UV divergent integrals appear. However, these are naturally handled by dimensional regularization, and renormalized by counter-terms in a diffeomorphism invariant fashion, see e.g. [31]. As it is also standard, we will set scaleless integrals to zero in the conservative sector, unless otherwise noted. This means we do not keep contributions in which propagators start and end on the same source (although in principle at a different times), as depicted in figure 1 , since these type of diagrams produce scaleless divergent contributions for potential modes. ${ }^{8}$ Moreover, unlike in the PN expansion, these diagrams cannot be connected to the second particle through worldline non-linearities using the Polyakov action. In general, only bulk graviton self-interaction are required at higher orders in $G$. Hence, for example, the entire set of diagrams that contribute to the effective action to $\mathcal{O}\left(G^{3}\right)$ are shown in figure 2 (plus mirror images). The diagrams (a) and (b) are the only two required to $\mathcal{O}\left(G^{2}\right)$. Furthermore, the one-point functions in diagrams (b), (c) and (d) are responsible for the Schwarzschild background to $\mathcal{O}\left(\left(G m_{2}\right)^{3}\right)$, such that only $(e),(f)$ and $(g)$ carry information beyond the test-particle limit.

As it turns out, many contributions to the effective action in the conservative sector are naturally reduced to two- and three-dimensional integrals. That is the case, as we shall see, due to the appearance of $\delta$ functions in the PM scheme that force the compo-

\footnotetext{
${ }^{8}$ As it is well known, hereditary radiative effects can also enter in the conservative sector [17, 28], which requires a careful study of IR/UV divergences and scaleless integrals [31]. That is partially due to the fact that radiation modes can generate non-zero contributions, e.g. $\left(x_{1}^{i} \dddot{x}_{1}^{j}\right)_{\mathrm{TF}}^{2}$ from the diagram figure 1a, and a tail-type correction from figure $1 \mathrm{~b}$, in the long-wavelength expansion [33]. We will not consider these contributions here and return to this issue in future work.
} 


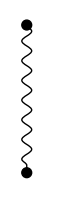

(a)

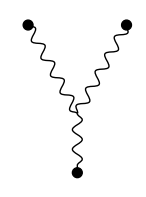

(b)

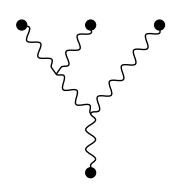

(c)

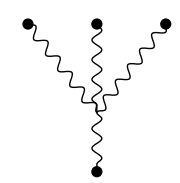

(d)

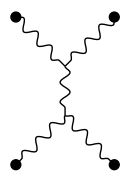

(e)

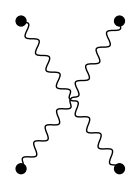

(f)

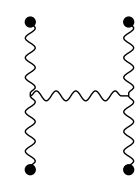

(g)

Figure 2. Feynman topologies needed for the computation of the effective action to $\mathcal{O}\left(G^{3}\right)$. The wavy line represents the propagator (or Green's function), while the black dots are the two worldlines at particle's 1 (bottom) and 2 (top), treated as external sources.

nent of the momentum to be orthogonal to the four velocities of the particles at infinity. (Something similar occurs in the derivation of the classical impulse starting from the scattering amplitude in [65].) However, for another class of diagrams isolating the conservative contribution to the effective action entails a prescription to perform the various $k_{1}^{0}, \ldots, k_{n}^{0}$ energy integrals that may appear at $n \mathrm{PM}$ order. We discuss the procedure below.

\subsection{Potential region}

The potential modes are natural objects in the PN framework. Contributions from the potential region in PN theory are obtained systematically by expanding the Feynman integrals in powers of $k_{0} /|\boldsymbol{k}|$. This is exploited in the EFT approach to reduce the complexity of the calculations, resorting to manifest power counting in the velocity expansion [8]. By splitting the four dimensional integrals into regions, the EFT framework has already achieved a high level of accuracy, at 4PN order [28, 30, 31] (and beyond [52, 53]). In this paper, however, we will not perform a small velocity expansion, and use the effective action to compute the scattering angle in relativistic two-body encounters instead. Therefore, our task here is to obtain the contribution from each diagram to the conservative sector in the PM expansion. Namely to incorporate the effects from potential modes at a given order in $G$, but to all orders in velocity. That is to say, we must use the relativistic version of the integrands and propagators, but only retain the contributions to the real part of the effective action which correspond to the conservative poles. In principle, once a contour in the $k^{0}$ complex plane is chosen, various poles may contribute to a given Feynman diagram. Therefore, in order to properly isolate the relevant (conservative) region of integration, we need a prescription to account for each one of them. We will adapt to our framework the procedure first introduced in [3], and later elaborated further in [63, 64, 90] to extend to higher PM orders.

In the EFT example we focus on in this paper, at 2PM, only one energy integral will be needed. As it was discussed in [3], in this case the procedure boils down to evaluating the $k^{0}$ energy integral by an (oriented) average over poles in the upper/lower half complex plane, that is

$$
\int \frac{\mathrm{d} k^{0}}{2 \pi}(\cdot)=\frac{i}{2}\left[\sum_{k_{\star}^{0} \in H^{+}} \underset{k^{0}=k_{\star}^{0}}{\operatorname{Res}}(\cdot)-\sum_{k_{\star}^{0} \in H^{-}} \operatorname{Res}_{k^{0}=k_{\star}^{0}}(\cdot)\right],
$$

while retaining only conservative contributions. This means we only keep the poles in the (potential) region $k^{0} \ll|\boldsymbol{k}|$. For our purposes here, the prescription in (3.15) will be sufficient. More generally, even though the poles in $[3,63,64]$ are different, e.g. no massive 
lines appear in the EFT computation, a given prescription can in principle be applied to any type of integral. Therefore, by adapting the rules from [64, 90] to our case we can evaluate all the energy integrals in the EFT approach at any PM order. For instance, by averaging over graviton permutations [126]. One of the basic tools is the identity [127]

$$
\delta\left(\sum_{i}^{n} \omega_{i}\right) \sum_{\text {Perms of } \omega_{i}} \frac{1}{\omega_{1}-i \epsilon} \cdots \frac{1}{\omega_{1}+\cdots+\omega_{n-1}-i \epsilon}=(2 \pi i)^{n-1} \prod_{i}^{n} \delta\left(\omega_{i}\right),
$$

to compute the energy integrals. For example, at 3PM order we may find integrands involving two energies, e.g.

$$
\frac{1}{k_{1}^{0}-i \epsilon} \frac{1}{k_{1}^{0}+k_{2}^{0}-i \epsilon}
$$

The idea is to re-write it using permutations of $k_{1}^{0}, k_{2}^{0}, k_{3}^{0}$ and imposing the condition $k_{3}^{0}+k_{2}^{0}+k_{1}^{0}=0$, such that

$$
\int \frac{\mathrm{d} k_{1}^{0}}{2 \pi} \frac{\mathrm{d} k_{2}^{0}}{2 \pi} \frac{1}{k_{1}^{0}-i \epsilon} \frac{1}{k_{1}^{0}+k_{2}^{0}-i \epsilon}=\frac{1}{3 !}(2 \pi i)^{2}=-\frac{2 \pi^{2}}{3} .
$$

The derivation of the dynamics to $\mathcal{O}\left(G^{3}\right)$ in the EFT approach is presented in [108].

Let us stress that, although the prescription to deal with energy integrals may turn out to be equivalent to the one in the amplitude program, the origin of the different classical contributions, as well as the associated integrals, will be quite different. For instance, we will not have to deal with super-classical IR divergences, as in [3, 63-65, 77]. (We do have UV poles, which are readily absorbed into counter-terms as we mentioned earlier.) We find, nonetheless, similarities with the calculations in [3, 63-65]. Notably, the result for the scattering angle to $2 \mathrm{PM}$ can be identified with a (classical) triangle integral, with vanishing contribution from the crossed-box. Yet, unlike what occurs in [3, 65, 77], the box diagram does not feature at all in the EFT computation. This is expected, since it would entail a singularity for which we would have no subtraction scheme, e.g. no EFT matching [3], nor 'cut diagram' [65], nor Born iterations [68, 77], as in methods dealing with the scattering amplitude. However, as we shall see in our example, the organization of the various terms entering in the final answer turn out to be remarkably distinct, as it was already seen in [65]. While this supports the complete independence in methodologies, it also begs for a deeper understanding of the nature of the different classical contributions at higher PM orders.

\subsection{Impulse \& deflection angle}

Using the effective action we can then derive the equations of motion from which we can compute the total momentum change, the impulse, as well as the scattering angle. Without loss of generality, let us study the deflection for particle 1. In the PM expansion the effective action takes the form, ${ }^{9}$

$$
S_{\text {eff }}=\sum_{n} \int \mathrm{d} \tau_{1} \mathcal{L}_{n}\left[x_{1}\left(\tau_{1}\right), x_{2}\left(\tau_{2}\right)\right]
$$

\footnotetext{
${ }^{9}$ At first glance, the resulting effective Lagrangian will appear to be non-local in time, involving an integral over $d \tau_{2}$. However, as we shall see, the resulting impulse and scattering angle in the conservative sector will be obtained from manifestly local interactions (as long as tail effects are ignored [28]).
} 
The $\mathcal{L}_{n}$ 's are the $\mathcal{O}\left(G^{n}\right)$ contributions to the effective Lagrangian obtained from the sum of all Feynman diagrams. The leading order (kinetic) term is given by, see (3.4),

$$
\mathcal{L}_{0}=-\frac{m_{1}}{2} \eta_{\mu \nu} v_{1}^{\mu}\left(\tau_{1}\right) v_{1}^{\nu}\left(\tau_{1}\right) .
$$

Performing the variation of the effective Lagrangian we obtain

$$
-\eta^{\mu \nu} \frac{\mathrm{d}}{\mathrm{d} \tau_{1}}\left(\frac{\partial \mathcal{L}_{0}}{\partial v_{1}^{\nu}}\right)=m_{1} \frac{\mathrm{d} v_{1}^{\mu}}{\mathrm{d} \tau_{1}}=-\eta^{\mu \nu}\left(\sum_{n=1}^{\infty} \frac{\partial \mathcal{L}_{n}}{\partial x_{1}^{\nu}\left(\tau_{1}\right)}-\frac{\mathrm{d}}{\mathrm{d} \tau_{1}}\left(\frac{\partial \mathcal{L}_{n}}{\partial v_{1}^{\nu}}\right)\right) .
$$

From here, and assuming $\mathcal{L}_{n \geq 1} \rightarrow 0$ at infinity, we find

$$
\Delta p_{1}^{\mu}=m_{1} \Delta v_{1}^{\mu}=-\eta^{\mu \nu} \sum_{n} \int_{-\infty}^{+\infty} \mathrm{d} \tau_{1} \frac{\partial \mathcal{L}_{n}}{\partial x_{1}^{\nu}},
$$

for the total change of four-momentum; or in 3D space,

$$
\Delta \boldsymbol{p}_{1}^{i}=-\Delta \boldsymbol{p}_{i 1}=\sum_{n} \int_{-\infty}^{+\infty} \mathrm{d} \tau_{1} \frac{\partial \mathcal{L}_{n}}{\partial \boldsymbol{x}_{1}^{i}} .
$$

Similarly for particle 2, which can also be obtained from momentum conservation.

The computation of the impulse then follows iteratively, order by order in the PM expansion. At leading order the trajectory is represented by a straight line, and higher PM effects result in a series of corrections, which we parameterize in terms of the proper time as follows $[65,93,94]$

$$
\begin{aligned}
& v_{a}^{\mu}\left(\tau_{1}\right)=u_{a}^{\mu}+\sum_{n} \delta^{(n)} v_{a}^{\mu}\left(\tau_{a}\right), \\
& x_{a}^{\mu}\left(\tau_{1}\right)=b_{a}^{\mu}+u_{a}^{\mu} \tau_{a}+\sum_{n} \delta^{(n)} x_{a}^{\mu}\left(\tau_{a}\right),
\end{aligned}
$$

where $u_{a}^{\mu}$ is the incoming velocity at infinity and $b_{a}^{\mu}=x_{a,(0)}^{\mu}-u_{a}^{\mu}\left(x_{a,(0)} \cdot u_{a}\right)$, with $x_{a,(0)}^{\mu}$ the initial position of each particle. For a scattering process we also have

$$
g_{\mu \nu}\left(x_{a}\left(\tau_{a}\right)\right) \rightarrow \eta_{\mu \nu}, \quad\left(\delta^{(n)} x_{a}^{\mu}\left(\tau_{a}\right), \delta^{(n)} v_{a}^{\mu}\left(\tau_{a}\right)\right) \rightarrow 0 \quad\left(\tau_{a} \rightarrow-\infty\right),
$$

such that the condition in (3.5) implies $u_{a}^{2}=1$, with $u_{1} \cdot u_{2}$ then identified with $\gamma$ in (2.5). Moreover, notice that $b_{a} \cdot u_{a}=0$, and the vector $b^{\mu} \equiv b_{2}^{\mu}-b_{1}^{\mu}$ will be thus associated with the impact parameter of the collision in the center-of-mass frame. In order to comply with standard literature we will incur in some minor abuse of notation and sometimes use $b$ to represent $\sqrt{-b^{\mu} b_{\mu}}$ in the final results, as well as for the four-vector appearing in dot products, e.g. $k \cdot b$, during the intermediate computations.

Due to the PM expansion in (3.24), there are various contributions to the impulse in (3.22), involving lower order terms evaluated on the deflected trajectories and expanded to the desired order, i.e.

$$
\Delta^{(n)} p_{a}^{\mu}=\sum_{k \leq n} \Delta_{\mathcal{L}_{k}}^{(n)} p_{a}^{\mu}
$$

with

$$
\Delta_{\mathcal{L}_{k}}^{(n)} p_{a}^{\mu} \equiv-\eta^{\mu \nu} \int_{-\infty}^{+\infty} \mathrm{d} \tau_{a}\left(\frac{\partial}{\partial x_{a}^{\nu}} \mathcal{L}_{k}\left[b_{a}+u_{a} \tau_{a}+\sum_{r=0}^{n-k} \delta^{(r)} x_{a}\right]\right)_{\mathcal{O}\left(G^{n}\right)}
$$


In summary, in order to compute the impulse we first derive the effective Lagrangian to $n$-th order via Feynman diagrams. We then extract the PM corrections to the equations of motion using the standard Euler-Lagrangian procedure. Hence, we insert the resulting trajectory into the time integral of the derivative of the effective Lagrangian with respect to the position, while keeping contributions up to the desired order. Given that for the impulse at $n \mathrm{PM}$ the deflection is needed to $(n-1) \mathrm{PM}$, we can proceed iteratively in powers of $G$, starting from the undeflected solution at $\mathcal{O}\left(G^{0}\right)$ in (3.24). Once the impulse is known we can then go to the center-of-mass frame and directly read off the scattering angle using, see e.g. [93],

$$
2 \sin \left(\frac{\chi}{2}\right)=\chi-\frac{1}{24} \chi^{3}+\mathcal{O}\left(\chi^{5}\right)=\frac{\left|\Delta \boldsymbol{p}_{1 \mathrm{~cm}}\right|}{p_{\infty}}=\frac{\sqrt{-\Delta p_{1}^{2}}}{p_{\infty}},
$$

where $p_{\infty}$ is given in (2.4). In the last equation we used that $\Delta p_{1}^{0}=0$ to re-write the expression in a covariant fashion using our conventions.

\section{Conservative binary dynamics to $2 \mathrm{PM}$}

In this section we compute all the dynamical invariants for bound orbits to $2 \mathrm{PM}$ order using the B2B map. We begin with the derivation of the scattering angle in the EFT approach, starting from the computation of the effective action. We obtain the (real part of the) effective Lagrangian following the same procedure as in NRGR, by 'integrating out' the metric degrees of freedom, while keeping the propagators fully relativistic. Therefore, unlike NRGR, we will not expand the propagators in powers of $k_{0} /|\boldsymbol{k}|$. We will handle the potential region as we described earlier in section 3.3. To perform the integrals we will often go to the frame where one of the incoming particles is initially at rest, and align the $x$-axis with the initial velocity of the companion. We will denote $\boldsymbol{k}_{\perp}$ the vectors in the orthogonal $z y$-plane.

\subsection{Effective Lagrangian}

\subsubsection{Tree}

The leading order contribution to the effective action comes from the 'tree' diagram in figure 2(a). The computation is straightforward, and we obtain

$$
\begin{aligned}
\mathcal{L}_{1} & =-i \frac{-i}{2} \frac{-i}{2} \int_{-\infty}^{+\infty} \mathrm{d} \tau_{2} \int_{k} \frac{i P_{\alpha \beta \mu \nu}}{k^{2}} v_{1}^{\alpha}\left(\tau_{1}\right) v_{1}^{\beta}\left(\tau_{1}\right) v_{2}^{\mu}\left(\tau_{2}\right) v_{2}^{\nu}\left(\tau_{2}\right) e^{i k \cdot\left(x_{1}\left(\tau_{1}\right)-x_{2}\left(\tau_{2}\right)\right)} \\
& =-\frac{m_{1} m_{2}}{8 M_{\mathrm{Pl}}^{2}} \int_{-\infty}^{+\infty} \mathrm{d} \tau_{2}\left(2\left(v_{1}\left(\tau_{1}\right) \cdot v_{2}\left(\tau_{2}\right)\right)^{2}-v_{1}^{2}\left(\tau_{1}\right) v_{2}^{2}\left(\tau_{2}\right)\right) \int_{k} \frac{1}{k^{2}} e^{i k \cdot\left(x_{1}\left(\tau_{1}\right)-x_{2}\left(\tau_{2}\right)\right)} .
\end{aligned}
$$

We can then read off the contribution to the equations of motion from the tree level action:

$$
\begin{aligned}
\frac{d v_{1}^{\mu}}{\mathrm{d} \tau_{1}}= & \frac{m_{2}}{8 M_{\mathrm{Pl}}^{2}} \int_{-\infty}^{\infty} \mathrm{d} \tau_{2}\left(2\left(v_{1}\left(\tau_{1}\right) \cdot v_{2}\left(\tau_{2}\right)\right)^{2}-v_{1}^{2}\left(\tau_{1}\right) v_{2}^{2}\left(\tau_{2}\right)\right) \int_{k} \frac{i k^{\mu}}{k^{2}} e^{i k \cdot\left(x_{1}\left(\tau_{1}\right)-x_{2}\left(\tau_{2}\right)\right)} \\
& -\frac{m_{2}}{4 M_{\mathrm{Pl}}^{2}} \int_{-\infty}^{\infty} \mathrm{d} \tau_{2}\left(2 v_{1}\left(\tau_{1}\right) \cdot v_{2}\left(\tau_{2}\right) v_{2}^{\mu}\left(\tau_{2}\right)-v_{2}^{2}\left(\tau_{2}\right) v_{1}^{\mu}\left(\tau_{1}\right)\right) \int_{k} \frac{i k \cdot v_{1}\left(\tau_{1}\right)}{k^{2}} e^{i k \cdot\left(x_{1}\left(\tau_{1}\right)-x_{2}\left(\tau_{2}\right)\right)} \\
& -\frac{m_{2}}{4 M_{\mathrm{Pl}}^{2}} \int_{-\infty}^{\infty} \mathrm{d} \tau_{2}\left(\left(2 \frac{\mathrm{d} v_{1}\left(\tau_{1}\right)}{\mathrm{d} \tau_{1}} \cdot v_{2}\left(\tau_{2}\right) v_{2}^{\mu}\left(\tau_{2}\right)-v_{2}^{2}\left(\tau_{2}\right) \frac{\mathrm{d} v_{1}^{\mu}\left(\tau_{1}\right)}{\mathrm{d} \tau_{1}}\right) \int_{k} \frac{1}{k^{2}} e^{i k \cdot\left(x_{1}\left(\tau_{1}\right)-x_{2}\left(\tau_{2}\right)\right)},\right.
\end{aligned}
$$


which will be useful later on to compute the deflection angle to second order in $G$. Notice the third line includes a term proportional to the acceleration, which can be treated systematically by replacing it with lower order equations of motion.

\subsubsection{One loop}

The effective action at $\mathcal{O}\left(G^{2}\right)$ (or 'one loop') has only one contribution, shown in the diagram in figure $2(\mathrm{~b})$. Using the cubic vertex described earlier we find

$$
\begin{aligned}
\mathcal{L}_{2}= & -\frac{m_{1} m_{2}^{2}}{16 M_{\mathrm{Pl}}^{3}} v_{1}^{\alpha}\left(\tau_{1}\right) v_{1}^{\beta}\left(\tau_{1}\right) \int \mathrm{d} \tau_{2} \int \mathrm{d} \tilde{\tau}_{2} v_{2}^{\gamma}\left(\tau_{2}\right) v_{2}^{\rho}\left(\tau_{2}\right) v_{2}^{\sigma}\left(\tilde{\tau}_{2}\right) v_{2}^{\kappa}\left(\tilde{\tau}_{2}\right) P_{\gamma \rho \tilde{\gamma} \tilde{\rho}}\left(k_{1}\right) P_{\sigma \kappa \tilde{\sigma} \tilde{\kappa}}\left(k_{2}\right) P_{\alpha \beta \tilde{\alpha} \tilde{\beta}}\left(k_{3}\right) \\
& \times \int_{k_{1,2,3}} e^{i k_{1} \cdot x_{1}\left(\tau_{1}\right)} e^{i k_{2} \cdot x_{2}\left(\tau_{2}\right)} e^{i k_{3} \cdot x_{2}\left(\tilde{\tau}_{2}\right)} \frac{V_{h h h}^{\tilde{\gamma} \tilde{\rho} \tilde{\kappa} \tilde{\alpha} \tilde{\beta} \tilde{\beta}}\left(k_{1}, k_{2}, k_{3}\right)}{k_{1}^{2} k_{2}^{2} k_{3}^{2}} \delta^{4}\left(k_{1}+k_{2}+k_{3}\right)+(1 \leftrightarrow 2), \quad(4.3)
\end{aligned}
$$

where

$$
\begin{aligned}
& i V_{h h h}^{a b c d e f}\left(p_{1}, p_{2}, p_{3}\right)=\frac{i}{4 M_{\mathrm{Pl}}} \times\left[4 p_{1} \cdot p_{2}\left(\eta^{a f} \eta^{b d} \eta^{c e}+\eta^{a e} \eta^{b d} \eta^{c f}\right)\right. \\
& +4 p_{2} \cdot p_{3}\left(\eta^{a e} \eta^{b c} \eta^{d f}+\eta^{a c} \eta^{b e} \eta^{d f}\right)+4 p_{1} \cdot p_{3}\left(\eta^{a d} \eta^{b f} \eta^{c e}+\eta^{a c} \eta^{b f} \eta^{d e}\right) \\
& +\left(p_{1} \cdot p_{2}+p_{2} \cdot p_{3}+p_{1} \cdot p_{3}\right)\left(\eta^{a b} \eta^{c d} \eta^{e f}-2 \eta^{a e} \eta^{b f} \eta^{c d}-2 \eta^{a b} \eta^{c e} \eta^{d f}-2 \eta^{a c} \eta^{b d} \eta^{e f}\right) \\
& -4 \eta^{a d} \eta^{c e} p_{1}^{f} p_{2}^{b}-4 \eta^{a e} \eta^{b c} p_{1}^{d} p_{2}^{f}+2 \eta^{a c} \eta^{b d}\left(p_{1}^{e} p_{2}^{f}+p_{1}^{f} p_{2}^{e}\right) \\
& -4 \eta^{a c} \eta^{d e} p_{2}^{f} p_{3}^{b}-4 \eta^{a e} \eta^{c f} p_{2}^{b} p_{3}^{d}+2 \eta^{c e} \eta^{d f}\left(p_{2}^{a} p_{3}^{b}+p_{2}^{b} p_{3}^{a}\right) \\
& \left.-4 \eta^{a f} \eta^{c e} p_{1}^{d} p_{3}^{b}-4 \eta^{a c} \eta^{b e} p_{1}^{f} p_{3}^{d}+2 \eta^{a e} \eta^{b f}\left(p_{1}^{c} p_{3}^{d}+p_{1}^{d} p_{3}^{c}\right)\right]
\end{aligned}
$$

Notice that, while a few terms are present at first, the orthogonality condition will drastically reduce the number of contributions once the diagram is evaluated on the undeflected solution. This turns out to be one of the major advantages of working with the scattering angle rather than computing the binding potential directly in a PM expansion.

\subsubsection{Trajectories}

To obtain the trajectories we must integrate the equations of motion using the expansion in (3.24). Since we restrict ourselves here to the impulse to $2 \mathrm{PM}$, we only need the first correction to the unperturbed solution. This follows from (4.2), which yields

$$
\begin{aligned}
\delta^{(1)} v_{1}^{\mu}\left(\tau_{1}\right) & =\frac{m_{2}}{4 M_{\mathrm{Pl}}^{2}}\left(\frac{2 \gamma^{2}-1}{2} \eta^{\mu \alpha}-\left(2 \gamma u_{2}^{\mu}-u_{1}^{\mu}\right) u_{1}^{\alpha}\right) \int_{-\infty}^{\tau_{1}} \mathrm{~d} \tilde{\tau}_{1} \int_{q} \hat{\delta}\left(q \cdot u_{2}\right) \frac{i q_{\alpha}}{q^{2}} e^{i\left(q \cdot u_{1}-i \epsilon\right) \tilde{\tau}_{1}} e^{i q \cdot b} \\
& =\frac{m_{2}}{4 M_{\mathrm{Pl}}^{2}}\left(\frac{2 \gamma^{2}-1}{2} \eta^{\mu \alpha}-\left(2 \gamma u_{2}^{\mu}-u_{1}^{\mu}\right) u_{1}^{\alpha}\right) \int_{q} \hat{\delta}\left(q \cdot u_{2}\right) \frac{i q_{\alpha}}{q^{2}} e^{i q \cdot b} \frac{(-i) e^{i\left(q \cdot u_{1}-i \epsilon\right) \tau_{1}}}{\left(q \cdot u_{1}-i \epsilon\right)}
\end{aligned}
$$

so that, after an additional time integration,

$$
\delta^{(1)} x_{1}^{\mu}\left(\tau_{1}\right)=-\frac{m_{2}}{4 M_{\mathrm{Pl}}^{2}}\left(\frac{2 \gamma^{2}-1}{2} \eta^{\mu \alpha}-\left(2 \gamma u_{2}^{\mu}-u_{1}^{\mu}\right) u_{1}^{\alpha}\right) \int_{q} \frac{i q_{\alpha} \hat{\delta}\left(q \cdot u_{2}\right)}{q^{2}\left(q \cdot u_{1}-i \epsilon\right)^{2}} e^{i q \cdot b} e^{i\left(q \cdot u_{1}-i \epsilon\right) \tau_{1}}
$$


We have added a factor of $-i \epsilon$ in all these expressions to ensure the convergence of the time integral at $\tilde{\tau} \rightarrow-\infty$, see e.g. [65]. Notice the resulting factor of $(q \cdot u-i \epsilon)^{-1}$ resembles the propagator in the heavy-quark effective theory (HQET) [128]. This will play an important role later on when we compute the impulse in the potential region, and is also directly associated to the large-mass limit of the scattering amplitude in $[3,77]$.

The deflection for the second particle can be obtained following the same procedure,

$$
\begin{aligned}
\delta^{(1)} v_{2}^{\mu}\left(\tau_{2}\right) & =\frac{m_{1}}{4 M_{\mathrm{Pl}}^{2}}\left(\frac{2 \gamma^{2}-1}{2} \eta^{\mu \alpha}-\left(2 \gamma u_{1}^{\mu}-u_{2}^{\mu}\right) u_{2}^{\alpha}\right) \int_{-\infty}^{\tau_{2}} \mathrm{~d} \tilde{\tau}_{2} \int_{q} \hat{\delta}\left(q \cdot u_{1}\right) \frac{-i q_{\alpha}}{q^{2}} e^{-i\left(q \cdot u_{2}+i \epsilon\right) \tilde{\tau}_{2}} e^{i q \cdot b} \\
& =\frac{m_{1}}{4 M_{\mathrm{Pl}}^{2}}\left(\frac{2 \gamma^{2}-1}{2} \eta^{\mu \alpha}-\left(2 \gamma u_{1}^{\mu}-u_{2}^{\mu}\right) u_{2}^{\alpha}\right) \int_{q} \hat{\delta}\left(q \cdot u_{1}\right) \frac{-i q_{\alpha}}{q^{2}} e^{i q \cdot b} \frac{(+i) e^{-i\left(q \cdot u_{2}+i \epsilon\right) \tau_{2}}}{\left(q \cdot u_{2}+i \epsilon\right)}(4.7)
\end{aligned}
$$

and

$$
\delta^{(1)} x_{2}^{\mu}\left(\tau_{2}\right)=\frac{m_{1}}{4 M_{\mathrm{Pl}}^{2}}\left(\frac{\left(2 \gamma^{2}-1\right)}{2} \eta^{\mu \alpha}-\left(2 \gamma u_{1}^{\mu}-u_{2}^{\mu}\right) u_{2}^{\alpha}\right) \int_{q} \frac{i q_{\alpha} \hat{\delta}\left(q \cdot u_{1}\right)}{q^{2}\left(q \cdot u_{2}+i \epsilon\right)^{2}} e^{i q \cdot b} e^{-i\left(q \cdot u_{2}+i \epsilon\right) \tau_{2}} .
$$

As expected, the PM shifts for particle 2 can be simply obtained from relabeling $1 \leftrightarrow 2$ in the corrections to particle 1, together with $q \rightarrow-q$. Notice, however, that because of our choice of signature, the factor of $\left(q \cdot u_{1}-i \epsilon\right)$ in (4.5) and (4.6) needed for the integral to converge turned into $\left(q \cdot u_{2}+i \epsilon\right)$. As we shall see, this distinction in the position of the poles will become relevant when combining all contributions to the impulse.

\subsection{Scattering angle}

We are now in position to compute the scattering angle for the two-body gravitational encounter. For the computation of the impulse we will concentrate on particle 1. A similar computation can be followed for particle 2 .

\subsubsection{Leading order impulse}

The leading order impulse follows from (3.22) applied to the 1PM effective action in (4.1) and evaluated in the undeflected trajectory in (3.24),

$$
\Delta_{\mathcal{L}_{1}}^{(1)} p_{1}^{\mu}=\frac{m_{1} m_{2}}{8 M_{\mathrm{Pl}}^{2}}\left(2 \gamma^{2}-1\right) \int_{k} i k^{\mu} \frac{\hat{\delta}\left(k \cdot u_{1}\right) \hat{\delta}\left(k \cdot u_{2}\right)}{k^{2}} e^{i k \cdot b} .
$$

To perform the integral we choose the frame at which particle 1 is initially at rest, $u_{1}=$ $(1,0,0,0)$. In such coordinates, the companion has four-velocity $u_{2}=(\gamma, \beta \gamma, 0,0)$, with $\beta \equiv \sqrt{\gamma^{2}-1} / \gamma$ the incoming relative velocity. Hence, using

$$
\int \frac{\mathrm{d}^{D} \boldsymbol{k}}{(2 \pi)^{D}} \frac{1}{\left(\boldsymbol{k}^{2}\right)^{n}} e^{-i \boldsymbol{k} \cdot \boldsymbol{x}}=\frac{1}{4^{n} \pi^{D / 2}} \frac{\Gamma[D / 2-n]}{\Gamma[n]}\left(\boldsymbol{x}^{2}\right)^{(-D / 2+n)},
$$

for the Fourier transform in $D=2$, we arrive at

$$
\begin{aligned}
\Delta_{\mathcal{L}_{1}}^{(1)} p_{1}^{\mu} & =-\frac{m_{1} m_{2}}{8 M_{\mathrm{Pl}}^{2}} \frac{\left(2 \gamma^{2}-1\right)}{\gamma \beta} \frac{\partial}{\partial \boldsymbol{b}_{\perp}} \int_{k_{\perp}} \frac{e^{-i \boldsymbol{k}_{\perp} \cdot \boldsymbol{b}_{\perp}}}{-\boldsymbol{k}_{\perp}^{2}}=-\frac{m_{1} m_{2}}{8 M_{\mathrm{Pl}}^{2}} \frac{\left(2 \gamma^{2}-1\right)}{\gamma \beta} \frac{\partial}{\partial \boldsymbol{b}_{\perp}}\left(+\frac{\log \left|\boldsymbol{b}_{\perp}\right|}{2 \pi}\right) \\
& =-2 m_{1} m_{2} G \frac{\left(2 \gamma^{2}-1\right)}{\sqrt{\gamma^{2}-1}} \frac{b^{\mu}}{\left|b^{2}\right|} .
\end{aligned}
$$


In the last line wrote the result in a covariant fashion using the impact parameter fourvector, $b^{\mu}$, orthogonal to the velocities, which in the rest frame of particle 1 it has components $b^{\mu}=\left(0, \boldsymbol{b}_{\perp}\right)$, obeying $\boldsymbol{b}_{\perp} \cdot \boldsymbol{u}_{2}=0$. From here, and using (3.28), we find

$$
\frac{\chi_{b}^{(1)}}{\Gamma}=\frac{\left(2 \gamma^{2}-1\right)}{\left(\gamma^{2}-1\right)}
$$

for the scattering angle at 1PM in impact parameter space, as expected.

\subsubsection{Next-to-leading order impulse}

According to (3.26), we have two contributions at NLO. We start with the 1PM action in (4.1) using the trajectory expanded to linear order in $G$. The two terms are due to the shifted position and velocity at $1 \mathrm{PM}$,

$$
\begin{array}{r}
\Delta_{\mathcal{L}_{1}}^{(2)} p_{1}^{\mu}=\frac{m_{1} m_{2}}{4 M_{\mathrm{Pl}}^{2}} \int_{-\infty}^{\infty} \mathrm{d} \tau_{1} \mathrm{~d} \tau_{2} \int_{k} \frac{i k^{\mu}}{k^{2}} e^{i k \cdot b+i k \cdot\left(u_{1} \tau_{1}-u_{2} \tau_{2}\right)}\left[\frac{\left(2 \gamma^{2}-1\right)}{2}(i k) \cdot\left(\delta^{(1)} x_{1}\left(\tau_{1}\right)-\delta^{(1)} x_{2}\left(\tau_{2}\right)\right)\right. \\
\left.+\left(2 \gamma u_{2 \beta}-u_{1 \beta}\right) \cdot \delta^{(1)} v_{1}^{\beta}\left(\tau_{1}\right)+\left(2 \gamma u_{1 \beta}-u_{2 \beta}\right) \cdot \delta^{(1)} v_{2}^{\beta}\left(\tau_{1}\right)\right],
\end{array}
$$

depending on both the deflection of particle 1 itself and the companion. Using an abuse of language, we refer to the contributions from particle 2 also as 'mirror images'. These terms are straightforward to compute by a simple relabeling. There is, however, one subtle point involving the factor of $q \cdot u_{1}-i \epsilon$ versus $q \cdot u_{2}+i \epsilon$ that appears in the deflection for particle 2 , and the integration over $q^{0}$. For for the sake of illustration, and simplicity, in what follows we will only refer to the effects due to the PM corrections to the motion of particle 1 itself, and deal with mirror images only when we combine all the intermediate results.

Inserting the values for the deflected velocity and position given in (4.5) and (4.6) into (4.13), and massaging the final expression, we have

$$
\Delta_{\mathcal{L}_{1}}^{(2)} p_{1}^{\mu}=i \frac{m_{1} m_{2}^{2}}{128 M_{\mathrm{Pl}}^{4}} \int_{k, \ell}\left[\left(2 \gamma^{2}-1\right)^{2} \ell^{2}-16 \gamma^{2}\left(k \cdot u_{1}\right)^{2}\right] \frac{\left(\ell^{\mu}-k^{\mu}\right) \hat{\delta}\left(k \cdot u_{2}\right) \hat{\delta}\left(\ell \cdot u_{2}\right) \hat{\delta}\left(\ell \cdot u_{1}\right)}{k^{2}(\ell-k)^{2}\left(k \cdot u_{1}-i \epsilon\right)^{2}} e^{i \ell \cdot b}
$$

where we have also discarded contributions which do not lead to a long-range interaction. We next move to the contribution from $\mathcal{L}_{2}$, which entails using the unperturbed trajectories in the result quoted in (4.3). As we discussed before, we will add the mirror image at the end. After performing the contractions, and retaining only terms which lead to a long-range interactions and do not vanish due to the $\delta$ functions, we arrive at the compact expression:

$$
\begin{aligned}
\Delta_{\mathcal{L}_{2}}^{(2)} p_{1}^{\mu}=i \frac{m_{1} m_{2}^{2}}{32 M_{\mathrm{Pl}}^{4}} \int_{k_{1,2}} \frac{k_{1}^{\mu} \hat{\delta}\left(k_{1} \cdot u_{1}\right) \hat{\delta}\left(k_{2} \cdot u_{2}\right) \hat{\delta}\left(k_{1} \cdot u_{2}\right)}{k_{1}^{2} k_{2}^{2}\left(k_{1}+k_{2}\right)^{2}} e^{i k_{1} \cdot b} \\
\quad \times\left(\gamma^{2} k_{1}^{2}+\left(k_{2} \cdot u_{1}\right)^{2}+\left(2 \gamma^{2}-1\right)\left(k_{1} \cdot k_{2}\right)\right) .
\end{aligned}
$$

To compute the integral we start by replacing $k_{1} \cdot k_{2}=\frac{1}{2}\left(\left(k_{1}+k_{2}\right)^{2}-k_{1}^{2}-k_{2}^{2}\right) \rightarrow-k_{1}^{2} / 2$, after noticing that is the only term leading to a long-range force. Furthermore, the $k_{2}$ - 
integral involving $\left(k_{2} \cdot u_{1}\right)^{2}$ can be simplified, using

$$
\begin{aligned}
\int_{k_{2}} \frac{\left(k_{2} \cdot u_{1}\right)^{2} \hat{\delta}\left(k_{2} \cdot u_{2}\right)}{k_{2}^{2}\left(k_{1}+k_{2}\right)^{2}} & =\frac{3}{8} \frac{\left(k_{1 \perp}^{\mu} k_{1 \perp}^{\nu}-\frac{1}{3}\left(\eta^{\mu \nu}-u_{2}^{\mu} u_{2}^{\nu}\right) k_{1 \perp}^{2}\right) u_{1}^{\mu} u_{1}^{\nu}}{\left(1-\frac{\left(k_{1} \cdot u_{2}\right)^{2}}{k_{1}^{2}}\right)^{2}} \frac{\hat{\delta}\left(k_{2} \cdot u_{2}\right)}{k_{2}} \\
& =\frac{k_{1}^{2}}{8}\left(\gamma^{2}-1\right) \int_{k_{2}} \frac{\hat{\delta}\left(k_{2} \cdot u_{2}\right)}{k_{2}^{2}\left(k_{1}+k_{2}\right)^{2}}+\mathcal{O}\left(k_{1} \cdot u_{a}\right),
\end{aligned}
$$

with $k_{1 \perp}^{\mu}=k_{1}^{\mu}-u_{2}^{\mu}\left(k_{1} \cdot u_{2}\right)$, and ignoring $k_{1} \cdot u_{a}$ factors due to the overall $\delta$ functions. Hence, after the innocuous re-labeling of momentum $k_{1}=\ell$ and $k_{2}=-k$,

$$
\Delta_{\mathcal{L}_{2}}^{(2)} p_{1}^{\mu}=i \frac{m_{1} m_{2}^{2}\left(\gamma^{2}+3\right)}{256 M_{\mathrm{Pl}}^{2}} \int_{k, \ell} \frac{\ell^{\mu} \hat{\delta}\left(\ell \cdot u_{1}\right) \hat{\delta}\left(\ell \cdot u_{2}\right) \hat{\delta}\left(k \cdot u_{2}\right)}{k^{2}(\ell-k)^{2}} e^{i \ell \cdot b} .
$$

Before we evaluate the result, which entails adding these two contributions and mirror images, it is instructive to split the two terms above into two other (more suggestive) contributions. Notice that the $\left(k \cdot u_{1}\right)^{2}$ in the numerator in (4.14) cancels out against the denominator. This allows us to complete the squares and replace the vector integral by

$$
\int_{k} \delta\left(k_{2} \cdot u_{2}\right) \frac{k^{\mu}}{k^{2}(\ell-k)^{2}}=\frac{\ell^{2}}{2 \ell_{\perp}^{2}} \int_{k} \delta\left(k_{2} \cdot u_{2}\right) \frac{\ell_{\perp}^{\mu}}{k^{2}(\ell-k)^{2}} \rightarrow \frac{1}{2} \int_{k} \delta\left(k_{2} \cdot u_{2}\right) \frac{\ell^{\mu}}{k^{2}(\ell-k)^{2}},
$$

where $\ell_{\perp}^{\mu} \equiv \ell^{\mu}-u_{2}^{\mu}\left(u_{2} \cdot \ell\right) \rightarrow \ell^{\mu}$, after setting $\ell \cdot u_{2}=0$ due to the overall $\delta$ functions. From here can then combine terms together and write the impulse at $2 \mathrm{PM}$, modulo mirror images,

$$
\Delta^{(2)} p_{1}^{\mu}=\Delta_{\triangle}^{(2)} p_{1}^{\mu}+\Delta_{u}^{(2)} p_{1}^{\mu}
$$

with

$$
\Delta_{\triangle}^{(2)} p_{1}^{\mu}=-\frac{3 m_{1} m_{2}^{2}\left(5 \gamma^{2}-1\right)}{256 M_{\mathrm{Pl}}^{4}} \frac{\partial}{\partial b_{\mu}} \int_{k, \ell} \frac{\hat{\delta}\left(k \cdot u_{2}\right) \hat{\delta}\left(\ell \cdot u_{2}\right) \hat{\delta}\left(\ell \cdot u_{1}\right)}{k^{2}(\ell-k)^{2}} e^{i \ell \cdot b}
$$

and

$$
\Delta_{u}^{(2)} p_{1}^{\mu}=i \frac{m_{1} m_{2}^{2}}{128 M_{\mathrm{Pl}}^{4}} \int_{k, \ell}\left(2 \gamma^{2}-1\right)^{2} \frac{\left(\ell^{\mu}-k^{\mu}\right) \ell^{2} \hat{\delta}\left(k \cdot u_{2}\right) \hat{\delta}\left(\ell \cdot u_{2}\right) \hat{\delta}\left(\ell \cdot u_{1}\right)}{k^{2}(\ell-k)^{2}\left(k \cdot u_{1}-i \epsilon\right)^{2}} e^{i \ell \cdot b} .
$$

Notice that the expression in (4.20) resembles the derivation of the impulse from the eikonal phase, see appendix A. To compute the integral we go to the rest frame of particle 2 this time, and using

$$
\int \frac{\mathrm{d}^{D} \boldsymbol{k}}{(2 \pi)^{D}} \frac{1}{\left((\boldsymbol{k}-\boldsymbol{p})^{2}\right)^{n_{1}}\left(\boldsymbol{k}^{2}\right)^{n_{2}}}=\frac{\Gamma\left[n_{1}+n_{2}-D / 2\right] \Gamma\left[D / 2-n_{1}\right] \Gamma\left[D / 2-n_{2}\right]}{\Gamma\left[n_{1}\right] \Gamma\left[n_{2}\right] \Gamma\left[D-n_{1}-n_{2}\right]} \frac{\left(\boldsymbol{p}^{2}\right)^{D / 2-n_{1}-n_{2}}}{(4 \pi)^{D / 2}},
$$

together with the Fourier transform in (4.10), we obtain

$$
\Delta_{\triangle}^{(2)} p_{1}^{\mu}=\frac{3 \pi}{4} \frac{\left(5 \gamma^{2}-1\right)}{\sqrt{\gamma^{2}-1}} \frac{\partial}{\partial \boldsymbol{b}_{\perp}}\left(\frac{G^{2} m_{2}^{2} m_{1}}{\left|\boldsymbol{b}_{\perp}\right|}\right)=-\frac{3 \pi m_{2}^{2} m_{1}}{4} \frac{\left(5 \gamma^{2}-1\right)}{\sqrt{\gamma^{2}-1}} \frac{G^{2} b^{\mu}}{\left|b^{2}\right|^{3 / 2}},
$$

where we wrote the result in a covariant fashion in terms of the impact parameter fourvector, $b^{\mu}$, which in the rest frame of particle 2 has components $b^{\mu}=\left(0, \boldsymbol{b}_{\perp}\right)$, with $\boldsymbol{b}_{\perp} \cdot \boldsymbol{u}_{1}=$ 
0. The other term in (4.21) is a little more involved, also when it comes to adding the contribution from the mirror image, as we show momentarily. It is convenient to first tensor-reduce the integral. Let us concentrate in the $\int_{q}$ part,

$$
\int_{q} \frac{\left(\ell^{\mu}-q^{\mu}\right) \hat{\delta}\left(q \cdot u_{2}\right)}{(\ell-q)^{2} q^{2}\left(q \cdot u_{1}-i \epsilon\right)^{2}}=A \ell^{\mu}+B\left(\gamma u_{2}^{\mu}-u_{1}^{\mu}\right)
$$

such that

$$
\Delta_{u}^{(2)} p_{1}^{\mu}=\frac{m_{1} m_{2}^{2}\left(2 \gamma^{2}-1\right)^{2}}{128 M_{\mathrm{Pl}}^{4}}\left[\int_{\ell}\left(i A(\ell, \gamma) \ell^{\mu}+i B(\ell, \gamma)\left(\gamma u_{2}^{\mu}-u_{1}^{\mu}\right)\right) \ell^{2} \hat{\delta}\left(\ell \cdot u_{2}\right) \hat{\delta}\left(\ell \cdot u_{1}\right) e^{i \ell \cdot b}\right],
$$

where we used that, because of all the $\delta$ functions involved, the result from dotting with $u_{2}$ must vanish. We multiply now by $\ell_{\mu}$, and use the same trick as before by writing $2 \ell \cdot q=\left(-(\ell-q)^{2}+\ell^{2}+q^{2}\right)$ to cancel local terms, so that

$$
A=\frac{1}{\ell^{2}} \int_{q} \frac{\left(\ell^{2}-(\ell \cdot q)\right) \hat{\delta}\left(q \cdot u_{2}\right)}{(\ell-q)^{2} q^{2}\left(q \cdot u_{1}-i \epsilon\right)^{2}}=\frac{1}{2} \int_{q} \frac{\hat{\delta}\left(q \cdot u_{2}\right)}{(\ell-q)^{2} q^{2}\left(q \cdot u_{1}-i \epsilon\right)^{2}} .
$$

For the $B$ coefficient we dot with $u_{1}$ instead. Using that $\ell \cdot u_{a}=0$, we find

$$
B=-\frac{1}{\gamma^{2}-1} \int_{q} \frac{\hat{\delta}\left(q \cdot u_{2}\right)}{(\ell-q)^{2} q^{2}\left(q \cdot u_{1}-i \epsilon\right)} .
$$

Notice that both these integrals, $A$ and $B$, resemble the crossed-box and triangle integrals in the large-mass limit of the one loop amplitude in $[3,63,64]$. To compute them we use the prescription we described in section 3.3. First of all, since we do not pick radiation poles, only the pole at $q \cdot u_{1}-i \epsilon=0$ contribute. Furthermore, notice that the $A$ integral converges, and that we have the two poles on the same side of the complex plane. By simply closing the contour in the opposite direction we can thus set $A=0$. This fact, which is equivalent to the vanishing of the crossed-box contribution at one loop [3, 63, 64], also explains why there is no $\left(2 \gamma^{2}-1\right)^{2}$ term in the scattering angle at $2 \mathrm{PM}^{10}{ }^{10}$ For the $B$ integral we find it convenient to move to the rest frame of particle 1 (recall $u_{1}=(1,0,0,0)$ and $\left.u_{2}=(\gamma, \gamma \beta, 0,0)\right)$. Then, using the prescription in (3.15) and averaging over conservative poles in the upper and lower complex plane, we have

$$
\begin{aligned}
i B & =-\frac{i}{\gamma^{2}-1} \int_{q} \frac{\mathrm{d} q^{0}}{2 \pi} \frac{\hat{\delta}\left(q \cdot u_{2}\right)}{(\ell-q)^{2} q^{2}\left(q^{0}-i \epsilon\right)}=-\frac{i}{\left(\gamma^{2}-1\right)} \frac{i}{2} \int_{q} \frac{\hat{\delta}\left(q \cdot u_{2}\right) \hat{\delta}\left(q^{0}\right)}{(\ell-q)^{2} q^{2}} \\
& =\frac{1}{8 \pi \gamma \beta\left(\gamma^{2}-1\right)} \frac{1}{\left(-\ell_{\perp}^{2}\right)}\left(\frac{1}{\bar{\epsilon}}+2 \log \left(-\ell_{\perp}^{2}\right)\right),
\end{aligned}
$$

where once again $\ell_{\perp}^{\mu}=\ell^{\mu}-\frac{u_{2}^{\mu}\left(\ell \cdot u_{2}\right)}{u_{2}^{2}}$, and we evaluated the integral in $D=4-2 \epsilon$ dimensions,

$$
\int_{q} \frac{\hat{\delta}\left(q \cdot u_{2}\right) \hat{\delta}\left(q^{0}\right)}{(\ell-q)^{2} q^{2}}=\frac{1}{4 \pi \gamma \beta} \frac{1}{\left(-\ell_{\perp}^{2}\right)}\left(\frac{1}{\bar{\epsilon}}+2 \log \left(-\ell_{\perp}^{2}\right)\right)
$$

\footnotetext{
${ }^{10}$ Notice that in $D>4$ the $A$ integral will give a non-zero contribution to the scattering angle, as it occurs also in the computation using the scattering amplitude [86].
} 
with some irrelevant factors absorbed into the definition of $\bar{\epsilon}$. As before, the $\delta$ functions in (4.25) will ultimately set $\ell_{\perp}^{2} \rightarrow \ell^{2}$, such that the contribution from the $1 / \bar{\epsilon}$ pole turns into a contact interaction that can be readily discarded in the classical limit. Therefore, only the logarithmic term survives as a long-range force. We expect this to be a generic feature also at higher PM orders, with intermediate spurious divergences producing analytic contributions, prior to the Fourier transform, which do not survive in the classical limit. (This is reminiscent of the small transfer momentum expansion of the scattering amplitude in $[3,63,64]$.) Finally, using

$$
\int_{\ell} \frac{\ell^{2}}{-\ell^{2}} \log \left(-\ell^{2}\right) e^{-i \ell \cdot b} \delta\left(\ell \cdot u_{1}\right) \delta\left(\ell \cdot u_{2}\right)=+\frac{1}{\pi \gamma \beta} \frac{1}{\left|\boldsymbol{b}_{\perp}\right|^{2}},
$$

we obtain

$$
\Delta_{u}^{(2)} p_{1}^{\mu}=2 \frac{m_{1} m_{2}^{2}\left(2 \gamma^{2}-1\right)^{2}}{\left(\gamma^{2}-1\right)^{2}} \frac{G^{2}}{\left|b^{2}\right|}\left(\gamma u_{2}^{\mu}-u_{1}^{\mu}\right),
$$

We now come to adding the contributions together and include also the mirror images. For the triangle term in (4.20) this is straightforward. First of all, by construction the terms from the total effective Lagrangian, prior to taking the partial derivative from (3.22), must be symmetric under $1 \leftrightarrow 2$. The same property translates to (4.20) before applying $\partial_{b_{\mu}}$, the action of which ultimately changes the relative sign with respect to the mirror image. The one associated with the term in (4.31) is a bit more subtle. It is easy to see that the main actor in (4.31) is the contribution from the $\eta^{\mu \alpha}$ term in (4.6). Notice that keeping $q$ unchanged while relabeling $1 \leftrightarrow 2$ results in an overall sign difference with respect to the same term in (4.8), which is then compensated by the relative sign in (4.13). Following similar steps we thus arrive at the same junctions, except that the deflected trajectory for particle 2 leads to poles at $q^{0}=-i \epsilon$ for the $A$ and $B$ integrals in (4.26) and (4.27). Hence, while the $A$ integral vanishes, when it comes to the $B$ integral the pole is now shifted to the lower complex half-plane, resulting in an overall minus sign. Therefore, the mirror image also leads to an anti-symmetrization in $1 \leftrightarrow 2$ in (4.31). At the end of the day, combining the terms and adding the $1 \mathrm{PM}$ result, we obtain

$$
\begin{aligned}
\Delta p_{1}^{\mu}= & -\frac{G m_{1} m_{2} b^{\mu}}{\left|b^{2}\right|}\left(\frac{2\left(2 \gamma^{2}-1\right)}{\sqrt{\gamma^{2}-1}}+\frac{3 \pi}{4} \frac{\left(5 \gamma^{2}-1\right)}{\sqrt{\gamma^{2}-1}} \frac{G M}{\left|b^{2}\right|^{1 / 2}}\right) \\
& +2 \frac{m_{1} m_{2}\left(2 \gamma^{2}-1\right)^{2}}{\left(\gamma^{2}-1\right)^{2}} \frac{G^{2}}{\left|b^{2}\right|}\left(\left(\gamma m_{2}+m_{1}\right) u_{2}^{\mu}-\left(\gamma m_{1}+m_{2}\right) u_{1}^{\mu}\right),
\end{aligned}
$$

for the total impulse to $2 \mathrm{PM}$ order in covariant form. As non-trivial check, notice that the expression in (4.32) is consistent with the on-shell condition,

$$
\left(p_{1}+\Delta p_{1}\right)^{2}=p_{1}^{2}, \quad 2 p_{1} \cdot \Delta p_{1}=-\Delta p_{1}^{2} .
$$

Moreover, because of the different PM orders involved, it is also straightforward to use the above equation, together with $\Delta p_{1}=-\Delta p_{2}$, to obtain the second term in (4.32) directly from the impulse in the $b^{\mu}$ direction. Furthermore, while the term involving the velocities was the most intricate of the two, because $b \cdot u_{a}=0$, it does not enter in the derivation of 
the $2 \mathrm{PM}$ angle. As a consequence, only the correction due to (4.23) matters to this order, yielding

$$
\frac{\chi_{b}^{(2)}}{\Gamma}=\frac{3 \pi}{8} \frac{\left(5 \gamma^{2}-1\right)}{\gamma^{2}-1}
$$

after using (3.28). The total impulse in (4.32) and resulting scattering angle are in agreement with the result obtained in [129].

\subsection{Adiabatic invariants}

Once the scattering angle is known, and translated from impact parameter to angular momentum space,

$$
\chi_{j}^{(1)}=\frac{\left(2 \gamma^{2}-1\right)}{\sqrt{\gamma^{2}-1}}, \chi_{j}^{(2)}=\frac{3 \pi}{8} \frac{\left(5 \gamma^{2}-1\right)}{\Gamma}
$$

we can then use (2.1) to reconstruct the B2B radial action via analytic continuation to $\mathcal{E}<0$,

$$
i_{r}^{2 \mathrm{PM}}(j, \mathcal{E})=\frac{\hat{p}_{\infty}}{\sqrt{-\hat{p}_{\infty}^{2}}} \chi_{j}^{(1)}(\mathcal{E})-j\left(1-\frac{2}{\pi} \frac{\chi_{j}^{(2)}(\mathcal{E})}{j^{2}}\right)=-j+\frac{\left(2 \gamma^{2}-1\right)}{\sqrt{1-\gamma^{2}}}+\frac{3}{4 j} \frac{\left(5 \gamma^{2}-1\right)}{\Gamma} .
$$

From here, following the B2B dictionary reviewed in section 2, we arrive at the same 2PM values for the gravitational observables for bound orbits derived in $[1,2]$ from the classical limit of the scattering amplitude/angle, namely

$$
\frac{\Delta \Phi_{2 \mathrm{PM}}}{2 \pi}=\frac{3}{4 j^{2}} \frac{\left(5 \gamma^{2}-1\right)}{\Gamma},
$$

for the periastron-advance, and

$$
\frac{T_{p}^{2 \mathrm{PM}}}{2 \pi G M}=\frac{\gamma\left(3-2 \gamma^{2}\right) \Gamma}{\left(1-\gamma^{2}\right)^{3 / 2}}+\frac{3}{4 j} \frac{15 \gamma^{2} \nu-10 \gamma(2 \nu-1)+\nu}{\Gamma^{2}},
$$

for the periastron-to-periastron period. From here we obtain for the azimuthal frequency,

$$
\begin{aligned}
G M \Omega_{\phi}^{2 \mathrm{PM}} & =\left(\frac{T_{p}^{2 \mathrm{PM}}}{2 \pi G M}\right)^{-1}\left(1+\frac{\Delta \Phi_{2 \mathrm{PM}}}{2 \pi}\right) \\
& =-\frac{\left(1-\gamma^{2}\right)^{3 / 2} \Gamma\left(4 j^{2} \Gamma+15 \gamma^{2}-3\right)}{j\left(4 j \gamma\left(2 \gamma^{2}-3\right) \Gamma^{3}-3\left(1-\gamma^{2}\right)^{3 / 2}(\nu+5 \gamma(2+(3 \gamma-4) \nu))\right)}
\end{aligned}
$$

It is also useful to notice that keeping only up to the 1PM coefficient of the scattering angle in the radial action leads to no periastron-advance, as expected, which implies [2]

$$
x_{1 \mathrm{PM}} \equiv\left(G M \Omega_{\phi}^{1 \mathrm{PM}}\right)^{2 / 3}=\left(\frac{T_{p}^{1 \mathrm{PM}}}{2 \pi G M}\right)^{-2 / 3}=\frac{\left(1-\gamma^{2}\right)}{\left(\left(3 \gamma-2 \gamma^{3}\right) \Gamma\right)^{2 / 3}} .
$$


We can also compute the redshift function, (re)obtaining at 2PM [2]

$$
\begin{aligned}
\left\langle z_{1}\right\rangle-\left\langle z_{1}^{(0)}\right\rangle= & \frac{\Gamma}{M(1+\Delta) \nu\left(4 j \gamma\left(3-2 \gamma^{2}\right) \Gamma^{3}+3\left(1-\gamma^{2}\right)^{3 / 2}(\nu+5 \gamma(2+(3 \gamma-4) \nu))\right)} \\
\times & {\left[8 j^{2}\left(1-\gamma^{2}\right)^{3 / 2} \Gamma \nu+4 j \gamma\left(2 \gamma^{2}-3\right) \Gamma(1+\Delta+2(\gamma-1) \nu)\right.} \\
& \left.+3\left(1-\gamma^{2}\right)^{3 / 2}\left(5 \gamma^{2}(\Delta-1) \nu-10 \gamma(1+\Delta-2 \nu)-(3+\Delta) \nu\right)\right],
\end{aligned}
$$

with

$$
\left\langle z_{1}^{(0)}\right\rangle=1+\frac{(1+\Gamma)(2 \nu+\Delta-1)}{2 \nu}
$$

and $\Delta \equiv \sqrt{1-4 \nu}$. The expression for $z_{2}$ results after replacing $\Delta \rightarrow-\Delta$.

These PM corrections include a series of PN terms (inside the $\gamma$ 's) at each order in $G$. As we discussed in $[1,2]$, the $2 \mathrm{PM}$ result for the periastron advance (and period) are one loop exact, in the sense that all the PN contributions at $1 / j^{2}$ (and $1 / j$ ) are encapsulated in the above expressions, to all orders in velocities. Moreover, as we emphasized in [1], the PN expansion of $x_{1 \mathrm{PM}}$ for circular orbits recovers the correct coefficient for all of the $\mathcal{O}\left(\mathcal{E}^{n} \nu^{n}\right)$ contributions at $n \mathrm{PN}$ order, for any value of $n$.

\subsubsection{Circular orbits}

To obtain the orbital frequency for circular orbits, we can proceed as explained in [1] by identifying the orbital elements, and then imposing the vanishing of the eccentricity. Or, equivalently, the simplicity of the radial action to $2 \mathrm{PM}$ allows us to also impose $i_{r}=0$, and then solve for $j$ as a function of $\gamma$ (see e.g. footnote 19 in [1]). Notice, however, in that case it is useful to use the 2PM-resummed version of the radial action [2],

$$
i_{r}^{2 \mathrm{PM}-\mathrm{res}}=\left(\frac{B}{\sqrt{-A}}-\sqrt{-C}\right)
$$

with

$$
\begin{aligned}
-A & =-\hat{p}_{\infty}^{2}=\frac{1-\gamma^{2}}{\Gamma^{2}}, \\
B & =\hat{p}_{\infty}^{2} \chi_{b}^{(1)}=\frac{2 \gamma^{2}-1}{\Gamma}, \\
-C & =j^{2}-\frac{4}{\pi} \hat{p}_{\infty}^{2} \chi_{b}^{(2)}=j^{2}\left(1-\frac{3\left(5 \gamma^{2}-1\right)}{2 \Gamma j^{2}}\right),
\end{aligned}
$$

yielding

$$
i_{r}^{2 \mathrm{PM}-\mathrm{res}}=\frac{\left(2 \gamma^{2}-1\right)}{\sqrt{1-\gamma^{2}}}-j \sqrt{1-\frac{3\left(5 \gamma^{2}-1\right)}{2 \Gamma j^{2}}} .
$$

Imposing the condition $i_{r}=0$ for a circular orbit, we then recover the value

$$
j_{2 \mathrm{PM}}^{2}=\frac{\left(2 \gamma^{2}-1\right)^{2}}{\left(1-\gamma^{2}\right)}+\frac{3\left(5 \gamma^{2}-1\right)}{2 \Gamma},
$$


for the angular momentum to 2PM [1]. We can then enter this expression in (4.39), resulting in the orbital frequency as a function of $\gamma$, and ultimately the binding energy from (2.5). A simpler route is to use the first law of black hole dynamics [122], and compute instead [1]

$$
\begin{aligned}
G M \Omega_{\text {circ }}^{2 \mathrm{PM}} & =\left(\Gamma \frac{\mathrm{d} j_{2 \mathrm{PM}}(\gamma)}{\mathrm{d} \gamma}\right)^{-1}=\frac{2 j_{2 \mathrm{PM}}}{\Gamma}\left(\frac{\mathrm{d} j_{2 \mathrm{PM}}^{2}(\gamma)}{\mathrm{d} \gamma}\right)^{-1} \\
& =\frac{2}{\Gamma} \sqrt{\frac{\left(2 \gamma^{2}-1\right)^{2}}{\left(1-\gamma^{2}\right)}+\frac{3\left(5 \gamma^{2}-1\right)}{2 \Gamma}}\left(\frac{3\left(-5 \gamma^{2} \nu+10 \gamma \Gamma^{2}+\nu\right)}{2 \Gamma^{3}}-\frac{2 \gamma\left(4 \gamma^{4}-8 \gamma^{2}+3\right)}{\left(\gamma^{2}-1\right)^{2}}\right)^{-1} .
\end{aligned}
$$

Notice that, keeping only the $1 \mathrm{PM}$ term, the variation in (2.8) that gives us the orbital period, $\partial_{\gamma} i_{r}$, is the same we obtain after solving for $j$ on orbits with $i_{r}=0$.

\subsection{Hamiltonian}

Due to the B2B map, we did not require a Hamiltonian in order to obtain all the gravitational observables in the conservative sector. However, as described in section 2, we can easily reconstruct it to derive also the equations of motion for bound orbits. Using the values for $\chi_{b}^{(1)}(E)$ and $\chi_{b}^{(2)}(E)$ in (4.12) and (4.34), and the expression in (2.21), we find

$$
\begin{aligned}
\frac{c_{1}\left(\boldsymbol{p}^{2}\right)}{1 !}=\frac{\nu^{2} M^{2}}{\Gamma^{2} \xi} & \left(1-2 \gamma^{2}\right), \\
\frac{c_{2}\left(\boldsymbol{p}^{2}\right)}{2 !}=\frac{\nu^{2} M^{3}}{\Gamma^{2} \xi}[ & {\left[\frac{3}{4}\left(1-5 \gamma^{2}\right)+\frac{\nu^{2}\left(2 \gamma^{2}-1\right)^{2}(3 \xi-1)}{2 \Gamma^{3} \xi^{2}}\right.} \\
& \left.+\frac{\left(2 \gamma^{2}-1\right)\left(4 \gamma \nu+\left(1-8 \gamma+6 \gamma^{2}\right) \nu^{2}\right)}{\Gamma^{3} \xi}\right],
\end{aligned}
$$

for the PM coefficients of the Hamiltonian, which we wrote using the convention of $[3,63$, 64]. It is straightforward to show that the Hamiltonian is equivalent to the one in $[3,63,64]$, e.g. eq. (10.10) of [64], written using a different combination for the two terms involving products of $1 \mathrm{PM}$ coefficients. Notice, nonetheless, that only the first terms in $c_{1}$ and $c_{2}$ actually matter, as illustrated by the radial action. The other two in $c_{2}$ are simply there to cancel the unwanted contributions which appear when deriving the $f_{1}, f_{2}$ in $(2.12)$ as a function of the energy. This feature is even more striking at higher PM orders, with the $\chi_{j}^{(2 n) \text { 's }}$ (and associated $f_{n}$ 's) carrying all the relevant information to compute dynamical invariants.

\section{$5 \quad$ Leading tidal effects}

In this section we provide an example of how finite-size effects are incorporated in our EFT approach for the PM regime. For concreteness, we study the correction due to the electricand magnetic-type tidal operators in the effective action, see (3.1) and (3.2),

$$
\sum_{a} c_{E^{2}}^{(a)} \int \mathrm{d} \tau_{a} E_{\mu \nu}\left(x_{a}\left(\tau_{a}\right)\right) E^{\mu \nu}\left(x_{a}\left(\tau_{a}\right)\right)+c_{B^{2}}^{(a)} \int \mathrm{d} \tau_{a} B_{\mu \nu}\left(x_{a}\left(\tau_{a}\right)\right) B^{\mu \nu}\left(x_{a}\left(\tau_{a}\right)\right),
$$

with $E_{\mu \nu}=R_{\mu \alpha \nu \beta} v^{\alpha} v^{\beta}$ and $B_{\mu \nu}=R_{\mu \alpha \nu \beta}^{\star} v^{\alpha} v^{\beta}$. In the weak field limit we have

$$
R_{a b c d}=\frac{1}{2}\left(\partial_{b} \partial_{c} h_{a d}+\partial_{a} \partial_{d} h_{b c}-\partial_{a} \partial_{c} h_{b d}-\partial_{b} \partial_{d} h_{a c}\right),
$$




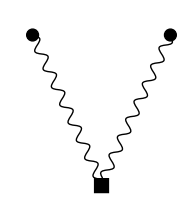

Figure 3. Feynman diagram with an insertion of a tidal operator represented by the square.

and therefore the contribution to the effective action is straightforward, and consists of computing the diagram shown in figure 3 , including the tidal operators and two mass insertions. Since we are interested in the leading PM correction to the impulse, only the undeflected worldlines in (3.24) are needed. The result for the effective action reads

$$
\begin{aligned}
\int \mathrm{d} \tau \mathcal{L}_{\mathrm{LO}}^{E^{2}}=\frac{c_{E^{2}}^{(1)} m_{2}^{2}}{64 M_{\mathrm{Pl}}^{4}} \int_{p, \ell} \frac{\hat{\delta}\left(p \cdot u_{2}\right)}{p^{2}(p-\ell)^{2}}\left(\left(1-2 \gamma^{2}\right)^{2}(p \cdot \ell)^{2}+2\left(1-4 \gamma^{2}\right)(p \cdot \ell)\left(p \cdot u_{1}\right)^{2}+2\left(p \cdot u_{1}\right)^{4}\right) \\
\times \hat{\delta}\left(\ell \cdot u_{1}\right) \hat{\delta}\left(\ell \cdot u_{2}\right) e^{i \ell \cdot b}+1 \leftrightarrow 2,
\end{aligned}
$$

for the electric-type, whereas the magnetic term we find

$$
\begin{gathered}
\int \mathrm{d} \tau \mathcal{L}_{\mathrm{LO}}^{B^{2}}=\frac{c_{B^{2}}^{(1)} m_{2}^{2}}{32 M_{\mathrm{Pl}}^{4}} \int_{p, \ell} \frac{\hat{\delta}\left(p \cdot u_{2}\right)}{p^{2}(p-\ell)^{2}}\left(2 \gamma^{2}\left(\gamma^{2}-1\right)(p \cdot \ell)^{2}+\left(1-4 \gamma^{2}\right)(p \cdot \ell)\left(p \cdot u_{1}\right)^{2}+\left(p \cdot u_{1}\right)^{4}\right) \\
\times \hat{\delta}\left(\ell \cdot u_{1}\right) \hat{\delta}\left(\ell \cdot u_{2}\right) e^{i \ell \cdot b}+1 \leftrightarrow 2 .
\end{gathered}
$$

As before, we compute the integral in the rest frame of particle 2, which reduces the integration in $p$ to $D=3$, and then use well-known results for the moments of the integral in (4.22), see e.g. [109]. After performing the final (Fourier transform) integral in $\ell$, we find

$$
\begin{aligned}
\int \mathrm{d} \tau \mathcal{L}_{\mathrm{LO}}^{E^{2}} & =\frac{9 \pi}{64} \frac{G^{2}}{\left|b^{2}\right|^{5 / 2}}\left(\frac{35 \gamma^{4}-30 \gamma^{2}+11}{\sqrt{\gamma^{2}-1}}\right)\left(c_{E^{2}}^{(1)} m_{2}^{2}+c_{E^{2}}^{(2)} m_{1}^{2}\right), \\
\int \mathrm{d} \tau \mathcal{L}_{\mathrm{LO}}^{B^{2}} & =\frac{9 \pi}{64} \frac{G^{2}}{\left|b^{2}\right|^{5 / 2}}\left(\frac{35 \gamma^{4}-30 \gamma^{2}-5}{\sqrt{\gamma^{2}-1}}\right)\left(c_{B^{2}}^{(1)} m_{2}^{2}+c_{B^{2}}^{(2)} m_{1}^{2}\right),
\end{aligned}
$$

for the electric- and magnetic-type contributions to the effective action, respectively. The impulse follows by taking a derivative with respect to the impact parameter. Using eq. (3.28) we thus get, in terms of the reduced angular momentum $(j=G M \mu J)$,

$$
\begin{aligned}
& \chi_{\mathrm{LO}}^{E^{2}}(j)=\frac{45 \pi \lambda_{E^{2}}}{64} \frac{\left(\gamma^{2}-1\right)^{2}\left(35 \gamma^{4}-30 \gamma^{2}+11\right)}{\Gamma^{5}} \frac{1}{j^{6}}, \\
& \chi_{\mathrm{LO}}^{B^{2}}(j)=\frac{45 \pi \lambda_{B^{2}}}{64} \frac{\left(\gamma^{2}-1\right)^{2}\left(35 \gamma^{4}-30 \gamma^{2}-5\right)}{\Gamma^{5}} \frac{1}{j^{6}},
\end{aligned}
$$

from tidal effects at leading PM order, with

$$
\lambda_{E^{2}} \equiv \frac{1}{G^{4} M^{5}}\left(c_{E^{2}}^{(1)} \frac{m_{2}}{m_{1}}+c_{E^{2}}^{(2)} \frac{m_{1}}{m_{2}}\right)
$$

and similarly for $\lambda_{B^{2}}$. The expressions in (5.7) and (5.8) agree with the result in [101], see e.g. eq. (6.2). Notice, as emphasised in [101], in the 'high energy limit' $\gamma \gg 1$ 
the scattering angle receives the same relative contribution from both the electric- and magnetic-type tidal effects. From (2.7) and (5.7) we obtain

$$
\frac{\Delta \Phi_{\mathrm{LO}}^{\text {Tidal }}}{2 \pi}=\frac{45\left(1-\gamma^{2}\right)^{2}}{64 \Gamma^{5} j^{6}}\left(\lambda_{E^{2}}\left(35 \gamma^{4}-30 \gamma^{2}+11\right)+\lambda_{B^{2}}\left(35 \gamma^{4}-30 \gamma^{2}-5\right)\right),
$$

for the periastron advance after analytic continuation in the binding energy to $\gamma<1$. This agrees with the Newtonian limit $[101]\left(\beta=\sqrt{1-\gamma^{2}} / \gamma \ll 1\right)$,

$$
\frac{\Delta \Phi_{\mathrm{LO}}^{\text {Tidal }}}{2 \pi}=\frac{45 \beta^{4}}{4 j^{6}}\left(\lambda_{E^{2}}+\mathcal{O}\left(\beta^{2}\right)\right)
$$

Once again, we can also reconstruct a Hamiltonian that includes tidal effect at leading PM order, but to all orders in the velocity. The derivation is straightforward using the recursion relation from [1], see section 2.3 , and we find

$$
\begin{aligned}
\frac{c_{6}}{6 !} & =-\frac{P_{6}(E)}{2 E \xi}+\cdots=-\frac{16 M^{12} \nu^{6} \chi_{j}^{(6)}(E)}{15 \pi E p_{\infty}^{4} \nu^{6} \xi}+\cdots=-\frac{16 M^{7} \Gamma^{3} \nu^{2} \chi_{j}^{(6)}(E)}{15 \pi \xi\left(1-\gamma^{2}\right)^{2}}+\cdots \\
& =-\frac{3 M^{7} \nu^{2}}{8 \Gamma^{2} \xi}\left(\lambda_{E^{2}}\left(35 \gamma^{4}-30 \gamma^{2}+11\right)+\lambda_{B^{2}}\left(35 \gamma^{4}-30 \gamma^{2}-5\right)\right)+\cdots
\end{aligned}
$$

such that

$$
\frac{H_{\mathrm{LO}}^{\mathrm{Tidal}}\left(\boldsymbol{p}^{2}\right)}{\mu}=-\frac{15 \nu}{8 \Gamma^{2} \xi}\left(\lambda_{E^{2}}\left(7 \gamma^{4}-6 \gamma^{2}+\frac{11}{5}\right)+\lambda_{B^{2}}\left(7 \gamma^{4}-6 \gamma^{2}-1\right)\right)\left(\frac{G M}{r}\right)^{6}
$$

at leading order in the PM expansion. Higher order corrections can be easily computed by including non-linear gravitational couplings as well as iterations from the modified worldlines.

\section{Discussion \& outlook}

Building upon NRGR [8] and the B2B map [1, 2], we developed a systematic framework to compute dynamical invariants for binary system in a PM expansion, to all orders in velocity. The two main ingredients in our formalism are: i) An EFT approach to compute the gravitational scattering angle in perturbation theory, and ii) The $\mathrm{B} 2 \mathrm{~B}$ radial action, constructed via analytic continuation from the scattering angle. We illustrated the procedure with two paradigmatic examples. First, we used the EFT formalism to compute the impulse and scattering angle in the conservative sector to $\mathcal{O}\left(G^{2}\right)$, and subsequently (re-)derived all the associated gravitational observables for bound orbits. Secondly, we computed the leading PM contribution due to tidal effects to the scattering angle and periastron advance. The results for the conservative dynamics to $2 \mathrm{PM}$ agree with the ones obtained through the $\mathrm{B} 2 \mathrm{~B}$ dictionary using the impetus formula $[1,2]$, applied to the classical limit of the one loop amplitude in [3]. For the sake of completeness, we reconstructed the Hamiltonian for the two-body dynamics from scattering data to NLO, which agrees with the one obtained in [3] via a matching calculation. We also computed the leading 
PM contribution to the Hamiltonian due to tidal effects, which is equivalent to the EOB approach discussed in [101].

One of the advantages of our formalism, in comparison with computing the (more intricate and gauge-dependent) gravitational potential in a PM expansion, is the dependence of the $\mathrm{B} 2 \mathrm{~B}$ radial action on the gauge-invariant, and much simpler, scattering angle instead. This feature notoriously simplifies the type of diagrams and integrals involved in our case; all the while incorporating the relativistic information that is lacking in a PN scheme (when both are kept up to the same order in $G$ ). Furthermore, although still relying on Feynman tools, the EFT approach circumvents somewhat the intermediate steps required, thus far, in the program to obtain classical gravitational physics out of quantum amplitudes [3, 62-91]. In particular, even though the impetus formula [1] removes the need of the additional matching calculation performed in [3, 62-64], or the intricate Born iterations of $[68,77]$, the mass-dependent loop integrals, and spurious IR super-classical divergences, still remain in the amplitude program. On the other hand, only massless integrals (without super-classical singularities) appear in the classical EFT framework. These differences are also manifest when computing gauge-invariant quantities, such as the scattering angle, as we demonstrated in the explicit example in this paper at $2 \mathrm{PM}$ order. Only two diagrams are needed for the NLO impulse, shown in figures $2 \mathrm{ab}$, and just the (Fourier transform of the) following integral,

$$
\int_{q} \frac{1}{q^{2}(q-\ell)^{2}}=\frac{1}{8|\ell|}
$$

was required when restricted to the scattering angle. Needless to say, the (super-classical) IR divergent box diagram never shows up, as expected. This is due to the lack of particle/anti-particle propagators yielding poles on opposite sides of the real axis. Moreover, the analogous to the crossed-box term, which appears here as a correction from the deflected trajectory in the tree level action, readily vanishes in the potential region. In practice, this means that the exact prescription to perform the energy integral(s) that we used in (4.27) was not needed to compute observables for bound orbits to 2PM. (That is the case because the extra term depending on the velocities in (4.32) is orthogonal to the impact parameter.) In contrast, even after removing/ignoring the box diagram by whichever method, the result using the scattering amplitude still requires the mass-dependent one loop triangle integral, with a contour prescription $[3,63,64,76]$ together with the largemass and small momentum-transfer limits. At the end of the day, as illustrated in [3, 65], the relevance of the integral in (6.1) emerges in the classical limit. (This is expected, since the massive field then collapses to a non-propagating source as in HQET [128].) Yet, in our approach, the resulting scattering angle and dynamical invariants to $2 \mathrm{PM}$ were obtained directly from (6.1). Moreover, in a classical EFT framework the large-mass limit is implicitly taken, and the small momentum-transfer expansion is equivalent to retaining only non-analytic terms prior to the Fourier transform, which yield long-range interactions. Notice this implied the removal of intermediate divergences that produce only contact terms, as in (4.27). The final answer for the scattering angle, however, emerged as a combination between a 1PM correction to the trajectory, due to the tree level effective action in figure $2 \mathrm{a}$, plus the leading one loop contribution from figure $2 \mathrm{~b}$ evaluated on the undeflected 
solution. This combination, also noted in [65], illustrates how the amplitude and classical derivations differ when it comes to the organization of all the relevant contributions. The reordering of terms suggests a dual understanding for the origin of the different pieces entering in the classical limit, which may ultimately help us also to incorporate powerful on-shell methods in our approach.

The EFT procedure in the PM expansion described here can be straightforwardly automatized to all orders. Since (for non-spinning bodies) worldline non-linearities are only produced by finite-size effects, the number of Feynman diagrams is relatively small when restricted to the monopole term, which notoriously simplifies the derivations. Moreover, for a large fraction of the diagrams evaluated on a straight worldline, which produce factors of $\delta\left(k \cdot u_{a}\right)$ after integration in the proper time, the calculation in the potential region resembles the same type of three-dimensional massless integrals that we find in NRGR with static propagators. The associated master integrals are known to four loops [29], akin of 5PM order. However, for another set of diagrams the associated $\delta$ functions yield new integrals which do not appear in the PN expansion. By replacing these $\delta$ 's by factors of $(q \cdot u-i \epsilon)^{-1}$, these turn out to be equivalent to the ones resulting from the iterations of the deflected worldlines in the computation of the impulse. As we emphasized, the type of integrals from this procedure resemble instead those of HQET, as it is the case for the NLO impulse in (4.27). Therefore, we expect the various powerful results in the literature, e.g. [109], can be then translated to our framework when pursuing higher PM orders. (Similar integrals appear in the soft expansion discussed in [90].) As we mentioned, notice that the term proportional to (4.27) does not enter in the scattering angle at 2PM. Furthermore, we can always resort to the on-shell condition in (4.33). This is remarkable feature will further simplify the computations at higher orders, by allowing us to restrict to derivation of the impulse to the $b^{\mu}$-direction.

The derivation of the 3PM impulse using the EFT formalism is reported in [108]. Notice, however, that the natural power counting in $1 / j$ of the B2B radial action in (2.1) requires the $4 \mathrm{PM}$ value as well. As we studied in [1,2], one can include all the contributions to $\chi_{j}^{(4)}$ from lower order terms in (2.16), i.e. using the impetus formula together with the results from $[63,64]$ to read off the value of the $f_{n \leq 3}$ 's, thus yielding a $2 \mathrm{PN}$ truncation to the radial action. Yet, as we emphasized [1,2], from the PM standpoint this truncation does not account for all the velocity contributions at $\mathcal{O}\left(1 / j^{3}\right)$, contrary to what happens at $\mathcal{O}(1 / j)$ with $f_{2}$ alone. ${ }^{11}$ Therefore, the scattering angle at $4 \mathrm{PM}$ remains a key ingredient to complete the next order in the $\mathrm{B} 2 \mathrm{~B}$ map that is yet to be calculated. The missing piece may be obtained from the classical limit of the three loop amplitude and/or the 4PM impulse; using the tools from $[3,63-65,76,77]$, the EFT framework developed here, or

\footnotetext{
${ }^{11}$ Regarding this point, in a recent paper [98] the authors claim that the radial action in $[1,2]$ truncated to 2PM, re-derived here in (4.36), is a "PN-incomplete 2PM truncation." It is straightforward to show that the expression given in [98] to $2 \mathrm{PM}, I_{r}^{\text {loc }}=-j+I_{0}^{S}+I_{1}^{S}(\gamma) /(h j)$ with $h=\Gamma$ and $I_{0}^{S}, I_{1}^{S}$ in their eqs. (9.3) and (9.5), coincides with (4.36). The derivation in [98] thus reproduces the $2 \mathrm{PM}$ result in [1, 2]. We think the authors of [98] perhaps meant the truncation with only the $f_{n \leq 3}$ contributions to $\chi_{j}^{(4)}$. As we emphasized in $[1,2]$, indeed this misses the (so far unknown) $f_{4}$ term. However, it is a consistent truncation as we showed explicitly by deriving all the observables to $3 \mathrm{PM} / 2 \mathrm{PN}$ in [2], including PN-exact terms controlled by (4.36).
} 
some hybrid approach between the two (plausibly in combination with the classical double copy [111-121], on-shell methods [103, 104], soft limits [90], and simplifications of the Einstein-Hilbert action [110]). In either case, the B2B dictionary [1, 2] provides a direct (analytic) connection between scattering data and bound states, neatly demonstrating the need to push the computations to (even) higher orders, to fully incorporate the power of the PM expansion in the dynamics of binary systems.

\section{Acknowledgments}

We thank the Munich Institute for Astro- and Particle Physics (MIAPP), supported by the DFG cluster of excellence "Origin and Structure of the Universe", and the participants of the program "Precision Gravity: From the LHC to LISA" for several fruitful discussions. In particular, Zvi Bern, Poul Damgaard, Guillaume Faye, Alfredo Guevara, Donal O'Connell, Radu Roiban, Chia-Hsien Shen, Mikhail Solon, Jan Steinhoff, and Justin Vines. We also thank Clifford Cheung, Zhengwen Liu and Julio Parra-Martinez for useful discussions. R.A.P. acknowledges financial support from the ERC Consolidator Grant "Precision Gravity: From the LHC to LISA" provided by the European Research Council (ERC) under the European Union's H2020 research and innovation programme (grant agreement No. 817791), as well as from the Deutsche Forschungsgemeinschaft (DFG, German Research Foundation) under Germany's Excellence Strategy (EXC 2121) 'Quantum Universe' (390833306). G.K. is supported by the Knut and Alice Wallenberg Foundation under grant KAW 2018.0441, and is supported in part by the US Department of Energy under contract DE-AC02-76SF00515.

\section{A Action \& impulse vs. amplitude \& eikonal}

In our derivation of the total impulse in (4.32) we found two contributions. One of them along the $b^{\mu}$ direction, reproduced here for the reader's convenience (with mirror image),

$$
\frac{3\left(1-5 \gamma^{2}\right)}{256 M_{\mathrm{Pl}}^{4}} \frac{\partial}{\partial b_{\mu}}\left[m_{1} m_{2}^{2} \int_{k, \ell} \frac{\hat{\delta}\left(k \cdot u_{2}\right) \hat{\delta}\left(\ell \cdot u_{2}\right) \hat{\delta}\left(\ell \cdot u_{1}\right)}{k^{2}(\ell-k)^{2}} e^{i \ell \cdot b}+1 \leftrightarrow 2\right],
$$

and another one proportional to the velocities. By inspection, the reader will notice the resemblance of the above equation with the eikonal approximation for the scattering amplitude, see e.g. [89]. Indeed, in the center-of-mass we have

$$
\Delta^{(2)} \boldsymbol{p}_{\perp}=\frac{1}{256 M_{\mathrm{Pl}}^{4}} \frac{3\left(5 \gamma^{2}-1\right)}{\sqrt{\gamma^{2}-1}} \frac{\partial}{\partial \boldsymbol{b}_{\perp}}\left[m_{1} m_{2}^{2} \int_{\boldsymbol{k}, \ell_{\perp}} \frac{1}{\boldsymbol{k}^{2}\left(\boldsymbol{\ell}_{\perp}-\boldsymbol{k}\right)^{2}} e^{-i \ell_{\perp} \cdot \boldsymbol{b}_{\perp}}+1 \leftrightarrow 2\right]=\frac{\partial}{\partial \boldsymbol{b}_{\perp}} \theta_{\text {eik }}^{(2)}
$$

with

$$
\theta_{\mathrm{eik}}^{(2)} \equiv \frac{\mu M^{2}}{256 M_{\mathrm{Pl}}^{4}} \frac{3\left(5 \gamma^{2}-1\right)}{\sqrt{\gamma^{2}-1}} \int_{\boldsymbol{k}, \ell_{\perp}} \frac{1}{\boldsymbol{k}^{2}\left(\boldsymbol{\ell}_{\perp}-\boldsymbol{k}\right)^{2}} e^{-i \ell_{\perp} \cdot \boldsymbol{b}_{\perp}}=\frac{3 \pi\left(5 \gamma^{2}-1\right)}{4 \sqrt{\gamma^{2}-1}} \frac{\mu(G M)^{2}}{\left|\boldsymbol{b}_{\perp}\right|} .
$$

It is then straightforward to extract the (IR-finite part of the) classical limit of the amplitude. Using the relationship,

$$
\theta_{\text {eik }}^{(2)}\left(\boldsymbol{b}_{\perp}\right)=\frac{1}{4 \mu M \sqrt{\gamma^{2}-1}} \int_{\boldsymbol{q}_{\perp}} \mathcal{M}_{\mathrm{cl}}^{(2)}\left(\boldsymbol{q}_{\perp}\right) e^{-i \boldsymbol{q}_{\perp} \cdot \boldsymbol{b}_{\perp}}
$$


we find

$$
\mathcal{M}_{\mathrm{cl}}^{(2)}(\boldsymbol{q})=\left(5 \gamma^{2}-1\right) \frac{6 \pi^{2} G^{2} \mu^{2} M^{3}}{|\boldsymbol{q}|},
$$

which agrees with the classical limit in $[3,63,64]$ (see e.g. first line of eq. 5.43 in [89]). In general, for contributions in the conservative sector, the impulse in the $b^{\mu}$ direction in the center-of-mass frame can always be written as

$$
\Delta p_{\perp}^{\mu}=\int_{\ell} M(\ell) \delta\left(\ell \cdot u_{1}\right) \delta\left(\ell \cdot u_{2}\right)\left(i \ell^{\mu}\right) e^{i \ell \cdot b} .
$$

The numerator, $M(\ell)$, results from 'loop-type' two-point functions with external (transfer) momentum $\ell$, as in (A.2). This expression suggest the definition

$$
S_{\mathrm{eik}}\left(\left|\boldsymbol{b}_{\perp}\right|, \gamma, m_{1}, m_{2}\right) \equiv \int_{\boldsymbol{\ell}_{\perp}} M\left(\boldsymbol{\ell}_{\perp}\right) e^{-i \boldsymbol{\ell}_{\perp} \cdot \boldsymbol{b}_{\perp}},
$$

such that

$$
\Delta \boldsymbol{p}_{\perp}=\frac{\partial}{\partial \boldsymbol{b}_{\perp}} S_{\mathrm{eik}}\left(\left|\boldsymbol{b}_{\perp}\right|, \gamma, m_{1}, m_{2}\right) .
$$

The reader will immediately inquire whether the $S_{\text {eik }}$, which coincides with the eikonal phase to $2 \mathrm{PM}, S_{\text {eik }}^{(2)}=\theta_{\text {eik }}^{(2)}$, is related to the classical action evaluated on the trajectories. This would not be entirely surprising, after all we can think of the scattering matrix in the classical limit as the exponential of the action on the classical solution, which would be captured by the eikonal approximation. As we know already, the Fourier transform of the amplitude encodes the value of the square of the momenta, through the impetus formula [1]. At the same time, from the radial action we have

$$
\frac{\chi+\pi}{2}=-\frac{1}{p_{\infty}} \frac{\partial}{\partial b} \int_{r_{\min }}^{\infty} p_{r}(r, b, E) \mathrm{d} r
$$

which combined with (A.8) and (3.28) implies

$$
S_{\text {eik }}=2\left(b \frac{\pi}{2}+\int_{r_{\min }}^{\infty} p_{r}(r, b, E) \mathrm{d} r\right),
$$

to one loop order. It is straightforward to show that $S_{\text {eik }}$ then coincides with (twice) the phase shift in the WKB/classical approximation, which in turn gives us the eikonal phase as we just discovered to $2 \mathrm{PM}$ order. At higher orders, however, the relationship is a bit more subtle, among other things because of the mismatch between the impact parameter entering in (A.8) and the one in the stationary phase approximation in the eikonal approach, e.g. [90].

We can, on the other hand, attempt to connect directly the impulse rather than the scattering angle. For instance, we know that on solution to the equations of motion, the derivative of the action w.r.t. the end point gives us the momenta at that point in time

$$
\boldsymbol{p}_{a}\left(\tau_{a}\right)=\frac{\partial S\left[x_{a}\right]}{\partial \boldsymbol{x}_{a}\left(\tau_{a}\right)} .
$$


Since at $\tau_{a} \rightarrow-\infty$ we have $\boldsymbol{p}_{a} \cdot \boldsymbol{b}_{\perp}=0$, the impulse along $b^{\mu}$ is determined by the limit

$$
\Delta \boldsymbol{p}_{a \perp}=\lim _{\tau_{a} \rightarrow \infty} \frac{\partial S\left[x_{a}\right]}{\partial \boldsymbol{x}_{a \perp}\left(\tau_{a}\right)} .
$$

Of course, on the undeflected trajectory, $\boldsymbol{x}_{a}=\boldsymbol{b}_{a}+\boldsymbol{u}_{a} \tau_{a}$, we find

$$
\Delta^{(1)} \boldsymbol{p}_{a \perp}=\frac{\partial S_{1}\left[x_{a}\right]}{\partial \boldsymbol{b}_{a}} .
$$

This tells us that, indeed, the function in (A.8) is the action at 1PM evaluated on the undeflected trajectory: $S_{\text {eik }}^{(1)}=S_{1}$. On the other hand, the solution is shifted at $2 \mathrm{PM}$, $\boldsymbol{b}_{a} \rightarrow \boldsymbol{b}_{a}+\delta^{(1)} \boldsymbol{x}_{a}$, with $\delta^{(1)} \boldsymbol{x}_{a} \cdot \boldsymbol{b}_{a} \neq 0$, which implies that the derivative in (A.8) and (A.12) are not the same, so that $S \neq S_{\text {eik }}$ beyond leading order. This can be seen explicitly in e.g. (4.13), where one notices that we can pull out the derivative w.r.t. the impact parameter, but only at the cost of adding a subtraction involving $\frac{\partial}{\partial \boldsymbol{b}} \delta^{(1)} \boldsymbol{x}_{a}$ (and also for the velocities). Hence, while from Lorentz invariance and the condition $b \cdot u_{a}=0$ we can always construct the function $S_{\text {eik }}$ in the conservative sector, the connection to the action evaluated on the solution is less direct. We will explore the properties of $S_{\text {eik }}$ in more detail elsewhere.

Open Access. This article is distributed under the terms of the Creative Commons Attribution License (CC-BY 4.0), which permits any use, distribution and reproduction in any medium, provided the original author(s) and source are credited.

\section{References}

[1] G. Kälin and R.A. Porto, From boundary data to bound states, JHEP 01 (2020) 072 [arXiv: 1910.03008] [INSPIRE].

[2] G. Kälin and R.A. Porto, From boundary data to bound states. Part II. Scattering angle to dynamical invariants (with twist), JHEP 02 (2020) 120 [arXiv:1911.09130] [INSPIRE].

[3] C. Cheung, I.Z. Rothstein and M.P. Solon, From scattering amplitudes to classical potentials in the post-Minkowskian expansion, Phys. Rev. Lett. 121 (2018) 251101 [arXiv: 1808.02489] [INSPIRE].

[4] LigO Scientific and Virgo collaborations, Open data from the first and second observing runs of advanced LIGO and advanced Virgo, arXiv:1912.11716 [INSPIRE].

[5] A. Buonanno and B.S. Sathyaprakash, Sources of gravitational waves: theory and observations, in General relativity and gravitation: a centennial perspective, Cambridge University Press, Cambridge, U.K. (2014), pg. 287 [arXiv:1410.7832] [INSPIRE].

[6] R.A. Porto, The tune of love and the nature(ness) of spacetime, Fortsch. Phys. 64 (2016) 723 [arXiv: 1606.08895 ] [INSPIRE].

[7] R.A. Porto, The music of the spheres: the dawn of gravitational wave science, arXiv: 1703.06440 [INSPIRE].

[8] W.D. Goldberger and I.Z. Rothstein, An effective field theory of gravity for extended objects, Phys. Rev. D 73 (2006) 104029 [hep-th/0409156] [InSPIRE].

[9] W.D. Goldberger, Les Houches lectures on effective field theories and gravitational radiation, in Les Houches summer school - Session 86. Particle physics and cosmology: the fabric of spacetime, (2007) [hep-ph/0701129] [INSPIRE]. 
[10] R.A. Porto and R. Sturani, Scalar gravity: post-Newtonian corrections via an effective field theory approach, in Les Houches summer school - session 86. Particle physics and cosmology: the fabric of spacetime, (2007) [gr-qc/0701105] [INSPIRE].

[11] S. Foffa and R. Sturani, Effective field theory methods to model compact binaries, Class. Quant. Grav. 31 (2014) 043001 [arXiv:1309.3474] [INSPIRE].

[12] I.Z. Rothstein, Progress in effective field theory approach to the binary inspiral problem, Gen. Rel. Grav. 46 (2014) 1726 [inSPIRE].

[13] V. Cardoso and R.A. Porto, Analytic approximations, perturbation theory, effective field theory methods and their applications, Gen. Rel. Grav. 46 (2014) 1682 [arXiv:1401.2193] [INSPIRE].

[14] R.A. Porto, The effective field theorist's approach to gravitational dynamics, Phys. Rept. 633 (2016) 1 [arXiv: 1601.04914] [INSPIRE].

[15] M. Beneke and V.A. Smirnov, Asymptotic expansion of Feynman integrals near threshold, Nucl. Phys. B 522 (1998) 321 [hep-ph/9711391] [INSPIRE].

[16] R.A. Porto, Lamb shift and the gravitational binding energy for binary black holes, Phys. Rev. D 96 (2017) 024063 [arXiv: 1703.06434] [INSPIRE].

[17] R.A. Porto and I.Z. Rothstein, Apparent ambiguities in the post-Newtonian expansion for binary systems, Phys. Rev. D 96 (2017) 024062 [arXiv:1703.06433] [INSPIRE].

[18] L. Blanchet, Gravitational radiation from post-Newtonian sources and inspiralling compact binaries, Living Rev. Rel. 17 (2014) 2 [arXiv:1310.1528] [INSPIRE].

[19] G. Schäfer and P. Jaranowski, Hamiltonian formulation of general relativity and post-Newtonian dynamics of compact binaries, Living Rev. Rel. 21 (2018) 7 [arXiv: 1805.07240] [INSPIRE].

[20] T. Damour, P. Jaranowski and G. Schäfer, Nonlocal-in-time action for the fourth post-Newtonian conservative dynamics of two-body systems, Phys. Rev. D 89 (2014) 064058 [arXiv: 1401.4548] [INSPIRE].

[21] P. Jaranowski and G. Schäfer, Derivation of local-in-time fourth post-Newtonian ADM Hamiltonian for spinless compact binaries, Phys. Rev. D 92 (2015) 124043 [arXiv: 1508.01016] [INSPIRE].

[22] L. Bernard, L. Blanchet, A. Bohé, G. Faye and S. Marsat, Fokker action of nonspinning compact binaries at the fourth post-Newtonian approximation, Phys. Rev. D 93 (2016) 084037 [arXiv: 1512.02876] [INSPIRE].

[23] L. Bernard, L. Blanchet, A. Bohé, G. Faye and S. Marsat, Dimensional regularization of the IR divergences in the Fokker action of point-particle binaries at the fourth post-Newtonian order, Phys. Rev. D 96 (2017) 104043 [arXiv: 1706. 08480] [INSPIRE].

[24] T. Marchand, L. Bernard, L. Blanchet and G. Faye, Ambiguity-free completion of the equations of motion of compact binary systems at the fourth post-Newtonian order, Phys. Rev. D 97 (2018) 044023 [arXiv: 1707.09289] [INSPIRE].

[25] J.B. Gilmore and A. Ross, Effective field theory calculation of second post-Newtonian binary dynamics, Phys. Rev. D 78 (2008) 124021 [arXiv:0810.1328] [INSPIRE].

[26] S. Foffa and R. Sturani, Effective field theory calculation of conservative binary dynamics at third post-Newtonian order, Phys. Rev. D 84 (2011) 044031 [arXiv:1104.1122] [INSPIRE]. 
[27] S. Foffa and R. Sturani, Dynamics of the gravitational two-body problem at fourth post-Newtonian order and at quadratic order in the Newton constant, Phys. Rev. D 87 (2013) 064011 [arXiv: 1206.7087] [INSPIRE].

[28] C.R. Galley, A.K. Leibovich, R.A. Porto and A. Ross, Tail effect in gravitational radiation reaction: time nonlocality and renormalization group evolution, Phys. Rev. D 93 (2016) 124010 [arXiv: 1511.07379] [INSPIRE].

[29] S. Foffa, P. Mastrolia, R. Sturani and C. Sturm, Effective field theory approach to the gravitational two-body dynamics, at fourth post-Newtonian order and quintic in the Newton constant, Phys. Rev. D 95 (2017) 104009 [arXiv:1612.00482] [INSPIRE].

[30] S. Foffa and R. Sturani, Conservative dynamics of binary systems to fourth post-Newtonian order in the EFT approach I: regularized Lagrangian, Phys. Rev. D 100 (2019) 024047 [arXiv: 1903.05113] [INSPIRE].

[31] S. Foffa, R.A. Porto, I. Rothstein and R. Sturani, Conservative dynamics of binary systems to fourth post-Newtonian order in the EFT approach II: renormalized Lagrangian, Phys. Rev. D 100 (2019) 024048 [arXiv: 1903.05118] [INSPIRE].

[32] W.D. Goldberger and I.Z. Rothstein, Dissipative effects in the worldline approach to black hole dynamics, Phys. Rev. D 73 (2006) 104030 [hep-th/0511133] [InSPIRE].

[33] W.D. Goldberger and A. Ross, Gravitational radiative corrections from effective field theory, Phys. Rev. D 81 (2010) 124015 [arXiv:0912.4254] [INSPIRE].

[34] A. Ross, Multipole expansion at the level of the action, Phys. Rev. D 85 (2012) 125033 [arXiv: 1202.4750] [INSPIRE].

[35] C.R. Galley and A.K. Leibovich, Radiation reaction at 3.5 post-Newtonian order in effective field theory, Phys. Rev. D 86 (2012) 044029 [arXiv: 1205.3842] [INSPIRE].

[36] A.K. Leibovich, N.T. Maia, I.Z. Rothstein and Z. Yang, Second post-Newtonian order radiative dynamics of inspiralling compact binaries in the effective field theory approach, Phys. Rev. D 101 (2020) 084058 [arXiv:1912.12546] [InSPIRE].

[37] R.A. Porto, Post-Newtonian corrections to the motion of spinning bodies in NRGR, Phys. Rev. D 73 (2006) 104031 [gr-qc/0511061] [INSPIRE].

[38] R.A. Porto and I.Z. Rothstein, The hyperfine Einstein-Infeld-Hoffmann potential, Phys. Rev. Lett. 97 (2006) 021101 [gr-qc/0604099] [INSPIRE].

[39] R.A. Porto, New results at $3 P N$ via an effective field theory of gravity, in $11^{\text {th }}$ Marcel Grossmann Meeting on General Relativity, World Scientific, Singapore (2007), pg. 2493 [gr-qc/0701106] [INSPIRE].

[40] R.A. Porto and I.Z. Rothstein, Comment on 'on the next-to-leading order gravitational $\operatorname{spin}(1)-\operatorname{spin}(2)$ dynamics' by J. Steinhoff et al., arXiv:0712.2032 [INSPIRE].

[41] R.A. Porto, Absorption effects due to spin in the worldline approach to black hole dynamics, Phys. Rev. D 77 (2008) 064026 [arXiv:0710.5150] [INSPIRE].

[42] R.A. Porto and I.Z. Rothstein, spin(1)spin(2) effects in the motion of inspiralling compact binaries at third order in the post-Newtonian expansion, Phys. Rev. D 78 (2008) 044012 [Erratum ibid. 81 (2010) 029904] [arXiv:0802.0720] [INSPIRE]. 
[43] R.A. Porto and I.Z. Rothstein, Next to leading order $\operatorname{spin}(1) \operatorname{spin}(1)$ effects in the motion of inspiralling compact binaries, Phys. Rev. D 78 (2008) 044013 [Erratum ibid. 81 (2010) 029905] [arXiv: 0804.0260] [INSPIRE].

[44] R.A. Porto, Next to leading order spin-orbit effects in the motion of inspiralling compact binaries, Class. Quant. Grav. 27 (2010) 205001 [arXiv:1005.5730] [INSPIRE].

[45] R.A. Porto, A. Ross and I.Z. Rothstein, Spin induced multipole moments for the gravitational wave flux from binary inspirals to third post-Newtonian order, JCAP 03 (2011) 009 [arXiv: 1007.1312] [INSPIRE].

[46] R.A. Porto, A. Ross and I.Z. Rothstein, Spin induced multipole moments for the gravitational wave amplitude from binary inspirals to 2.5 post-Newtonian order, JCAP 09 (2012) 028 [arXiv: 1203.2962] [INSPIRE].

[47] N.T. Maia, C.R. Galley, A.K. Leibovich and R.A. Porto, Radiation reaction for spinning bodies in effective field theory I: spin-orbit effects, Phys. Rev. D 96 (2017) 084064 [arXiv: 1705.07934] [INSPIRE].

[48] N.T. Maia, C.R. Galley, A.K. Leibovich and R.A. Porto, Radiation reaction for spinning bodies in effective field theory II: spin-spin effects, Phys. Rev. D 96 (2017) 084065 [arXiv: 1705. 07938] [INSPIRE].

[49] M. Levi and J. Steinhoff, Complete conservative dynamics for inspiralling compact binaries with spins at fourth post-Newtonian order, arXiv:1607.04252 [INSPIRE].

[50] M. Levi, A.J. Mcleod and M. Von Hippel, $N^{3} L O$ gravitational spin-orbit coupling at order $G^{4}$, arXiv:2003.02827 [INSPIRE].

[51] M. Levi, A.J. Mcleod and M. Von Hippel, NNNLO gravitational quadratic-in-spin interactions at the quartic order in $G$, arXiv:2003.07890 [INSPIRE].

[52] S. Foffa, P. Mastrolia, R. Sturani, C. Sturm and W.J. Torres Bobadilla, Static two-body potential at fifth post-Newtonian order, Phys. Rev. Lett. 122 (2019) 241605 [arXiv: 1902.10571] [INSPIRE].

[53] J. Blümlein, A. Maier and P. Marquard, Five-loop static contribution to the gravitational interaction potential of two point masses, Phys. Lett. B 800 (2020) 135100 [arXiv: 1902.11180] [INSPIRE].

[54] S. Foffa and R. Sturani, Hereditary terms at next-to-leading order in two-body gravitational dynamics, Phys. Rev. D 101 (2020) 064033 [arXiv:1907.02869] [INSPIRE].

[55] L. Blanchet, S. Foffa, F. Larrouturou and R. Sturani, Logarithmic tail contributions to the energy function of circular compact binaries, Phys. Rev. D 101 (2020) 084045 [arXiv: 1912.12359] [INSPIRE].

[56] S. Foffa, Gravitating binaries at $5 P N$ in the post-Minkowskian approximation, Phys. Rev. D 89 (2014) 024019 [arXiv:1309.3956] [INSPIRE].

[57] Y. Iwasaki, Quantum theory of gravitation vs. classical theory - fourth-order potential, Prog. Theor. Phys. 46 (1971) 1587 [InSPIRE].

[58] J.J.M. Carrasco, Gauge and gravity amplitude relations, in Theoretical Advanced Study Institute in Elementary Particle Physics. Journeys through the precision frontier: amplitudes for colliders, World Scientific, Singapore (2015), pg. 477 [arXiv:1506.00974] [INSPIRE]. 
[59] H. Elvang and Y.-T. Huang, Scattering amplitudes in gauge theory and gravity, Cambridge University Press, Cambridge, U.K. (2015).

[60] C. Cheung, TASI lectures on scattering amplitudes, in Proceedings, Theoretical Advanced Study Institute in Elementary Particle Physics: anticipating the next discoveries in particle physics (TASI 2016), Boulder, CO, U.S.A., 6 June-1 July 2016, R. Essig and I. Low eds., World Scientific, Singapore (2018), pg. 571 [arXiv: 1708.03872] [INSPIRE].

[61] Z. Bern, J.J. Carrasco, M. Chiodaroli, H. Johansson and R. Roiban, The duality between color and kinematics and its applications, arXiv:1909.01358 [INSPIRE].

[62] D. Neill and I.Z. Rothstein, Classical space-times from the S matrix, Nucl. Phys. B 877 (2013) 177 [arXiv: 1304.7263] [INSPIRE].

[63] Z. Bern, C. Cheung, R. Roiban, C.-H. Shen, M.P. Solon and M. Zeng, Scattering amplitudes and the conservative Hamiltonian for binary systems at third post-Minkowskian order, Phys. Rev. Lett. 122 (2019) 201603 [arXiv: 1901.04424] [INSPIRE].

[64] Z. Bern, C. Cheung, R. Roiban, C.-H. Shen, M.P. Solon and M. Zeng, Black hole binary dynamics from the double copy and effective theory, JHEP 10 (2019) 206 [arXiv: 1908.01493] [INSPIRE].

[65] D.A. Kosower, B. Maybee and D. O'Connell, Amplitudes, observables, and classical scattering, JHEP 02 (2019) 137 [arXiv:1811.10950] [INSPIRE].

[66] B. Maybee, D. O'Connell and J. Vines, Observables and amplitudes for spinning particles and black holes, JHEP 12 (2019) 156 [arXiv:1906.09260] [INSPIRE].

[67] C.R. Galley and R.A. Porto, Gravitational self-force in the ultra-relativistic limit: the "large- $N$ " expansion, JHEP 11 (2013) 096 [arXiv: 1302.4486] [INSPIRE].

[68] B.R. Holstein and A. Ross, Spin effects in long range gravitational scattering, arXiv:0802.0716 [INSPIRE].

[69] N.E.J. Bjerrum-Bohr, J.F. Donoghue and P. Vanhove, On-shell techniques and universal results in quantum gravity, JHEP 02 (2014) 111 [arXiv:1309.0804] [INSPIRE].

[70] V. Vaidya, Gravitational spin Hamiltonians from the S matrix, Phys. Rev. D 91 (2015) 024017 [arXiv: 1410 . 5348] [INSPIRE].

[71] A. Guevara, Holomorphic classical limit for spin effects in gravitational and electromagnetic scattering, JHEP 04 (2019) 033 [arXiv:1706.02314] [INSPIRE].

[72] M.-Z. Chung, Y.-T. Huang, J.-W. Kim and S. Lee, The simplest massive S-matrix: from minimal coupling to black holes, JHEP 04 (2019) 156 [arXiv:1812.08752] [INSPIRE].

[73] A. Guevara, A. Ochirov and J. Vines, Scattering of spinning black holes from exponentiated soft factors, JHEP 09 (2019) 056 [arXiv:1812.06895] [INSPIRE].

[74] S. Caron-Huot and Z. Zahraee, Integrability of black hole orbits in maximal supergravity, JHEP 07 (2019) 179 [arXiv: 1810.04694] [INSPIRE].

[75] A. Guevara, A. Ochirov and J. Vines, Black-hole scattering with general spin directions from minimal-coupling amplitudes, Phys. Rev. D 100 (2019) 104024 [arXiv:1906.10071] [INSPIRE].

[76] N.E.J. Bjerrum-Bohr, P.H. Damgaard, G. Festuccia, L. Planté and P. Vanhove, General relativity from scattering amplitudes, Phys. Rev. Lett. 121 (2018) 171601 [arXiv: 1806. 04920] [INSPIRE]. 
[77] A. Cristofoli, N.E.J. Bjerrum-Bohr, P.H. Damgaard and P. Vanhove, Post-Minkowskian Hamiltonians in general relativity, Phys. Rev. D 100 (2019) 084040 [arXiv:1906.01579] [INSPIRE].

[78] N. Arkani-Hamed, Y.-T. Huang and D. O'Connell, Kerr black holes as elementary particles, JHEP 01 (2020) 046 [arXiv:1906.10100] [InSPIRE].

[79] N.E.J. Bjerrum-Bohr, A. Cristofoli and P.H. Damgaard, Post-Minkowskian scattering angle in Einstein gravity, JHEP 08 (2020) 038 [arXiv:1910.09366] [INSPIRE].

[80] M.-Z. Chung, Y.-T. Huang and J.-W. Kim, Classical potential for general spinning bodies, JHEP 09 (2020) 074 [arXiv: 1908.08463] [INSPIRE].

[81] Y.F. Bautista and A. Guevara, From scattering amplitudes to classical physics: universality, double copy and soft theorems, arXiv:1903.12419 [INSPIRE].

[82] Y.F. Bautista and A. Guevara, On the double copy for spinning matter, arXiv:1908.11349 [INSPIRE].

[83] A. Koemans Collado, P. Di Vecchia and R. Russo, Revisiting the second post-Minkowskian eikonal and the dynamics of binary black holes, Phys. Rev. D 100 (2019) 066028 [arXiv: 1904.02667] [INSPIRE].

[84] H. Johansson and A. Ochirov, Double copy for massive quantum particles with spin, JHEP 09 (2019) 040 [arXiv: 1906.12292] [INSPIRE].

[85] R. Aoude, K. Haddad and A. Helset, On-shell heavy particle effective theories, JHEP 05 (2020) 051 [arXiv: 2001.09164] [INSPIRE].

[86] A. Cristofoli, P.H. Damgaard, P. Di Vecchia and C. Heissenberg, Second-order post-Minkowskian scattering in arbitrary dimensions, JHEP 07 (2020) 122 [arXiv: 2003. 10274] [INSPIRE].

[87] M.-Z. Chung, Y.-T. Huang, J.-W. Kim and S. Lee, Complete Hamiltonian for spinning binary systems at first post-Minkowskian order, JHEP 05 (2020) 105 [arXiv:2003.06600] [INSPIRE].

[88] Z. Bern, H. Ita, J. Parra-Martinez and M.S. Ruf, Universality in the classical limit of massless gravitational scattering, Phys. Rev. Lett. 125 (2020) 031601 [arXiv:2002.02459] [INSPIRE].

[89] Z. Bern, A. Luna, R. Roiban, C.-H. Shen and M. Zeng, Spinning black hole binary dynamics, scattering amplitudes and effective field theory, arXiv:2005.03071 [INSPIRE].

[90] J. Parra-Martinez, M.S. Ruf and M. Zeng, Extremal black hole scattering at $\mathcal{O}\left(G^{3}\right)$ : graviton dominance, eikonal exponentiation, and differential equations, JHEP 11 (2020) 023 [arXiv: 2005. 04236] [INSPIRE].

[91] A. Antonelli, A. Buonanno, J. Steinhoff, M. van de Meent and J. Vines, Energetics of two-body Hamiltonians in post-Minkowskian gravity, Phys. Rev. D 99 (2019) 104004 [arXiv: 1901.07102] [INSPIRE].

[92] T. Damour and A. Nagar, The effective-one-body approach to the general relativistic two body problem, Lect. Notes Phys. 905 (2016) 273 [INSPIRE].

[93] T. Damour, Gravitational scattering, post-Minkowskian approximation and effective one-body theory, Phys. Rev. D 94 (2016) 104015 [arXiv:1609.00354] [INSPIRE]. 
[94] T. Damour, High-energy gravitational scattering and the general relativistic two-body problem, Phys. Rev. D 97 (2018) 044038 [arXiv:1710.10599] [InSPIRE].

[95] D. Bini, T. Damour and A. Geralico, Novel approach to binary dynamics: application to the fifth post-Newtonian level, Phys. Rev. Lett. 123 (2019) 231104 [arXiv:1909.02375] [INSPIRE].

[96] T. Damour, Classical and quantum scattering in post-Minkowskian gravity, Phys. Rev. D 102 (2020) 024060 [arXiv: 1912.02139] [INSPIRE].

[97] D. Bini, T. Damour and A. Geralico, Binary dynamics at the fifth and fifth-and-a-half post-Newtonian orders, Phys. Rev. D 102 (2020) 024062 [arXiv:2003.11891] [INSPIRE].

[98] D. Bini, T. Damour and A. Geralico, Sixth post-Newtonian local-in-time dynamics of binary systems, Phys. Rev. D 102 (2020) 024061 [arXiv: 2004.05407] [InSPIRE].

[99] J. Vines, J. Steinhoff and A. Buonanno, Spinning-black-hole scattering and the test-black-hole limit at second post-Minkowskian order, Phys. Rev. D 99 (2019) 064054 [arXiv: 1812.00956] [INSPIRE].

[100] N. Siemonsen and J. Vines, Test black holes, scattering amplitudes and perturbations of Kerr spacetime, Phys. Rev. D 101 (2020) 064066 [arXiv: 1909.07361] [InSPIRE].

[101] D. Bini, T. Damour and A. Geralico, Scattering of tidally interacting bodies in post-Minkowskian gravity, Phys. Rev. D 101 (2020) 044039 [arXiv:2001.00352] [InSPIRE].

[102] T. Damour, P. Jaranowski and G. Schäfer, Dynamical invariants for general relativistic two-body systems at the third post-Newtonian approximation, Phys. Rev. D 62 (2000) 044024 [gr-qc/9912092] [INSPIRE].

[103] Z. Bern, L.J. Dixon, D.C. Dunbar and D.A. Kosower, One loop $n$ point gauge theory amplitudes, unitarity and collinear limits, Nucl. Phys. B 425 (1994) 217 [hep-ph/9403226] [INSPIRE].

[104] Z. Bern, L.J. Dixon, D.C. Dunbar and D.A. Kosower, Fusing gauge theory tree amplitudes into loop amplitudes, Nucl. Phys. B 435 (1995) 59 [hep-ph/9409265] [INSPIRE].

[105] Z. Bern, J.J.M. Carrasco and H. Johansson, New relations for gauge-theory amplitudes, Phys. Rev. D 78 (2008) 085011 [arXiv:0805.3993] [INSPIRE].

[106] Z. Bern, J.J.M. Carrasco and H. Johansson, Perturbative quantum gravity as a double copy of gauge theory, Phys. Rev. Lett. 105 (2010) 061602 [arXiv:1004.0476] [INSPIRE].

[107] C. Cheung and M.P. Solon, Classical gravitational scattering at $\mathcal{O}\left(G^{3}\right)$ from Feynman diagrams, JHEP 06 (2020) 144 [arXiv:2003.08351] [INSPIRE].

[108] G. Kälin, Z. Liu and R.A. Porto, Conservative dynamics of binary systems to third post-Minkowskian order from the effective field theory approach, arXiv:2007.04977 [INSPIRE].

[109] V.A. Smirnov, Analytic tools for Feynman integrals, Springer Tracts Mod. Phys. 250 (2012) [INSPIRE].

[110] C. Cheung and G.N. Remmen, Hidden simplicity of the gravity action, JHEP 09 (2017) 002 [arXiv: 1705. 00626] [INSPIRE].

[111] R. Monteiro, D. O'Connell and C.D. White, Black holes and the double copy, JHEP 12 (2014) 056 [arXiv: 1410.0239] [INSPIRE]. 
[112] A. Luna et al., Perturbative spacetimes from Yang-Mills theory, JHEP 04 (2017) 069 [arXiv: 1611.07508] [INSPIRE].

[113] W.D. Goldberger and A.K. Ridgway, Radiation and the classical double copy for color charges, Phys. Rev. D 95 (2017) 125010 [arXiv:1611.03493] [InSPIRE].

[114] W.D. Goldberger and A.K. Ridgway, Bound states and the classical double copy, Phys. Rev. D 97 (2018) 085019 [arXiv: 1711.09493] [INSPIRE].

[115] J. Li and S.G. Prabhu, Gravitational radiation from the classical spinning double copy, Phys. Rev. D 97 (2018) 105019 [arXiv: 1803.02405] [InSPIRE].

[116] C.-H. Shen, Gravitational radiation from color-kinematics duality, JHEP 11 (2018) 162 [arXiv: 1806. 07388] [INSPIRE].

[117] J. Plefka, J. Steinhoff and W. Wormsbecher, Effective action of dilaton gravity as the classical double copy of Yang-Mills theory, Phys. Rev. D 99 (2019) 024021 [arXiv: 1807.09859] [INSPIRE].

[118] J. Plefka, C. Shi, J. Steinhoff and T. Wang, Breakdown of the classical double copy for the effective action of dilaton-gravity at NNLO, Phys. Rev. D 100 (2019) 086006 [arXiv: 1906. 05875] [INSPIRE].

[119] K. Kim, K. Lee, R. Monteiro, I. Nicholson and D. Peinador Veiga, The classical double copy of a point charge, JHEP 02 (2020) 046 [arXiv: 1912.02177] [INSPIRE].

[120] W.D. Goldberger and J. Li, Strings, extended objects, and the classical double copy, JHEP 02 (2020) 092 [arXiv: 1912.01650] [INSPIRE].

[121] L. Alfonsi, C.D. White and S. Wikeley, Topology and Wilson lines: global aspects of the double copy, JHEP 07 (2020) 091 [arXiv: 2004.07181] [INSPIRE].

[122] A. Le Tiec, L. Blanchet and B.F. Whiting, The first law of binary black hole mechanics in general relativity and post-Newtonian theory, Phys. Rev. D 85 (2012) 064039 [arXiv:1111.5378] [INSPIRE].

[123] O.B. Firsov, Determination of the forces acting between atoms using the differential effective cross-section for elastic scattering, Zh. Eksp. Teor. Fiz. 24 (1953) 279.

[124] A. Kuntz, Half-solution to the two-body problem in general relativity, Phys. Rev. D 102 (2020) 064019 [arXiv: 2003.03366] [inSPIRE].

[125] S. Rafie-Zinedine, Simplifying quantum gravity calculations, master's thesis, Lund U., Lund, Sweden (2018) [arXiv:1808.06086] [INSPIRE].

[126] R. Akhoury, R. Saotome and G. Sterman, High energy scattering in perturbative quantum gravity at next to leading power, arXiv:1308.5204 [INSPIRE].

[127] R. Saotome and R. Akhoury, Relationship between gravity and gauge scattering in the high energy limit, JHEP 01 (2013) 123 [arXiv:1210.8111] [INSPIRE].

[128] B. Grinstein, Lectures on heavy quark effective theory, in Workshop on high-energy phenomenology (CINVESTAV), (1991), pg. 0161 [INSPIRE].

[129] K. Westpfahl, High-speed scattering of charged and uncharged particles in general relativity, Fortsch. Phys. 33 (1985) 417 [INSPIRE]. 